\title{
Research Article \\ Searching for the Minimal Seesaw Models at the LHC and Beyond
}

\author{
Arindam Das \\ School of Physics, KIAS, Seoul 130-722, Republic of Korea \\ Correspondence should be addressed to Arindam Das; dasarindamphysics@gmail.com
}

Received 28 July 2017; Accepted 21 February 2018; Published 19 April 2018

Academic Editor: Ning Chen

Copyright ( 2018 Arindam Das. This is an open access article distributed under the Creative Commons Attribution License, which permits unrestricted use, distribution, and reproduction in any medium, provided the original work is properly cited. The publication of this article was funded by SCOAP . $^{3}$

\begin{abstract}
The existence of the tiny neutrino mass and the flavor mixing can be naturally explained by type I Seesaw model which is probably the simplest extension of the Standard Model (SM) using Majorana type SM gauge singlet heavy Right Handed Neutrinos (RHNs). If the RHNs are around the Electroweak- (EW-) scale having sizable mixings with the SM light neutrinos, they can be produced at the high energy colliders such as Large Hadron Collider (LHC) and future $100 \mathrm{TeV}$ proton-proton (pp) collider through the characteristic signatures with the same-sign dilepton introducing lepton number violations (LNV). On the other hand Seesaw models, namely, inverse Seesaw, with small LNV parameter can accommodate EW-scale pseudo-Dirac neutrinos with sizable mixings with SM light neutrinos while satisfying the neutrino oscillation data. Due to the smallness of the LNV parameter of such models, the "smoking-gun" signature of same-sign dilepton is suppressed where the RHNs in the model will be manifested at the LHC and future $100 \mathrm{TeV}$ pp collider dominantly through the Lepton number conserving (LNC) trilepton final state with Missing Transverse Energy (MET). Studying various production channels of such RHNs, we give an updated upper bound on the mixing parameters of the light-heavy neutrinos at the $13 \mathrm{TeV} \mathrm{LHC}$ and future $100 \mathrm{TeV}$ pp collider.
\end{abstract}

\section{Introduction}

The experimental evidence of the neutrino oscillation and flavor mixings from neutrino oscillation experiments [1-6] indicates that the SM is not enough to explain the existence of the tiny neutrino mass and flavor mixing. After the pioneering discovery of the $d=5$ operator [7] within the SM using the SM leptons, SM Higgs doublets, and $\Delta L=2(L=$ Lepton number) unit of LNV, it turned out that the Seesaw mechanism [8-14] could be the simplest idea to explain the small neutrino mass and flavor mixing where the SM can be extended by the SM-gauge singlet Majorana type RHNs. After the Electroweak (EW) symmetry breaking, the light Majorana neutrino masses are generated after the so-called type I Seesaw mechanism.

In type I Seesaw, we introduce SM gauge-singlet Majorana RHNs $N_{R}^{\beta}$ where $\beta$ is the flavor index. $N_{R}^{\beta}$ couples with the SM lepton doublets $\ell_{L}^{\alpha}$ and the SM Higgs doublet $H$. The relevant part of the Lagrangian is

$$
\mathscr{L} \supset-Y_{D}^{\alpha \beta} \overline{\ell_{L}^{\alpha}} H N_{R}^{\beta}-\frac{1}{2} m_{N}^{\alpha \beta} \overline{N_{R}^{\alpha C}} N_{R}^{\beta}+\text { H.c., }
$$

where $Y_{D}^{\alpha \beta}$ is the Yukawa coupling, $\ell_{L}^{\alpha}$ is the SM $S U(2)_{L}$ lepton doublet, and $m_{N}$ is the Majorana mass term. After the spontaneous EW symmetry breaking by the vacuum expectation value (VEV), $H=\left(\begin{array}{c}v / \sqrt{2} \\ 0\end{array}\right)$, we obtain the Dirac mass matrix as $M_{D}=Y_{D} v / \sqrt{2}$ suppressing the indices. Using the Dirac and Majorana mass matrices we can write the neutrino mass matrix as

$$
M_{\nu}=\left(\begin{array}{cc}
0 & M_{D} \\
M_{D}^{T} & m_{N}
\end{array}\right),
$$

where $M_{D}$ is the Dirac mass matrix and $m_{N}$ is the Majorana mass term. Diagonalizing $M_{v}$ we obtain the Seesaw formula for the light Majorana neutrinos as

$$
m_{\nu} \simeq-M_{D} m_{N}^{-1} M_{D}^{T}
$$


For $m_{N} \sim 100 \mathrm{GeV}$, we may find $Y_{D} \sim 10^{-6}$ with $m_{\nu} \sim 0.1 \mathrm{eV}$. However, in the general parameterization for the Seesaw formula [15], $Y_{D}$ can be large and sizable $(\mathcal{O}(1))$, and this is the case we consider in this paper.

The searches of the Majorana RHNs can be performed by the "smoking-gun" of the same-sign dilepton plus di-jet signal which is suppressed by the square of the light-heavy neutrino mixing parameter $\left|V_{\ell N}\right|^{2} \simeq\left|m_{D} m_{N}^{-1}\right|^{2}$. A comprehensive general study on the parameters of $\left|V_{\ell N}\right|^{2}$ has been given in [16] using the data from the neutrino oscillation experiments [1-6], bounds from the Lepton Flavor Violation (LFV) experiments [17-19], Large Electron-Positron (LEP), and Electroweak Precision test [20-27] experiments using the nonunitarity effects $[28,29]$ applying the Casas-Ibarra conjecture [15, 30-33]. At this point we mention that [16] has a good agreement with a previous analysis [34] on the sterile neutrinos. The bounds in [16] have been compared with the existing results in $[23,26,27,35-37]$ considering the degenerate Majorana RHNs. In case of Seesaw mechanism the Dirac Yukawa matrix $\left(Y_{D}\right)$ can carry the flavors where the RHN mass matrix is considered to be diagonal. This case is favored by the neutrino oscillation data as studied in $[16,30,38-42]$. Such a scenario for the Seesaw scenario is called the Flavor Nondiagonal (FND). The other possibility of considering both of the diagonal $Y_{D}$ and $m_{N}$ is not supported by the neutrino oscillation data.

Since any number of singlets can be added in a gauge theory without contributing to anomalies, one can utilize such freedom to find a natural alternative of the lowscale realization of the Seesaw mechanism. Simplest among such scenarios is commonly known as the inverse Seesaw mechanism $[43,44]$ where a small Majorana neutrino mass originates from tiny LNV parameters rather than being suppressed by the RHN mass as done in the case of conventional Seesaw mechanism. In the inverse Seesaw model two sets of SM-singlet RHNs are introduced which are pseudo-Dirac by nature and their Dirac Yukawa couplings can be even order one, while reproducing the neutrino oscillation data. Therefore, at the high energy colliders such pseudo-Dirac neutrinos can be produced through a sizable mixing with the SM light neutrinos [45-52].

In the inverse Seesaw mechanism the relevant part of the Lagrangian is given by

$$
\mathscr{L} \supset-Y_{D}^{\alpha \beta} \overline{\ell_{L}^{\alpha}} H N_{R}^{\beta}-m_{N}^{\alpha \beta} \overline{S_{L}^{\alpha}} N_{R}^{\beta}-\frac{1}{2} \mu_{\alpha \beta} \overline{S_{L}^{\alpha}} S_{L}^{\beta^{C}}+\text { H.c., }
$$

where $N_{R}^{\alpha}$ and $S_{L}^{\beta}$ are two SM-singlet heavy neutrinos with the same lepton numbers, $m_{N}$ is the Dirac mass matrix, and $\mu$ is a small Majorana mass matrix violating the lepton numbers. After the EW symmetry breaking we obtain the neutrino mass matrix as

$$
M_{\nu}=\left(\begin{array}{ccc}
0 & M_{D} & 0 \\
M_{D}^{T} & 0 & m_{N}^{T} \\
0 & m_{N} & \mu
\end{array}\right) .
$$

Diagonalizing this mass matrix we obtain the light neutrino mass matrix

$$
M_{\nu} \simeq M_{D} m_{N}^{-1} \mu\left(m_{N}^{-1}\right)^{T} M_{D}^{T}
$$

Note that the smallness of the light neutrino mass originates from the small LNV term $\mu$. The smallness of $\mu$ allows the $m_{D} m_{N}^{-1}$ parameter to be on the order one even for an EW scale RHNs. In the inverse Seesaw case we can consider $Y_{D}$ as nondiagonal when $\mu$ and $m_{N}$ are diagonal which is called the Flavor Nondiagonal (FND) case. On the other hand we can also consider the diagonal $Y_{D}, m_{N}$ when $\mu$ will be nondiagonal. This situation is called the Flavor Democratic scenario. For the inverse Seesaw both of the FND and FD cases are supported by the neutrino oscillation data. In this article we will consider the FND case from the Seesaw and the FD case from the inverse Seesaw mechanisms.

Through the Seesaw mechanism, a flavor eigenstate $(\nu)$ of the SM neutrino is expressed in terms of the mass eigenstates of the light $\left(v_{m}\right)$ and heavy $\left(N_{m}\right)$ Majorana neutrinos such as

$$
\nu \simeq v_{m}+V_{\ell N} N_{m} .
$$

Using the mass eigenstates, the charged current (CC) interaction for the heavy neutrino is given by

$$
\mathscr{L}_{\mathrm{CC}} \supset-\frac{g}{\sqrt{2}} W_{\mu} \bar{e} \gamma^{\mu} P_{L} V_{\ell N} N_{m}+\text { H.c., }
$$

where $e$ denotes the three generations of the charged leptons in the vector form, and $P_{L}=(1 / 2)\left(1-\gamma_{5}\right)$ is the projection operator. Similarly, the neutral current (NC) interaction is given by

$$
\begin{gathered}
\mathscr{L}_{\mathrm{NC}} \supset-\frac{g}{2 c_{w}} Z_{\mu}\left[\overline{N_{m}} \gamma^{\mu} P_{L}\left|V_{\ell N}\right|^{2} N_{m}\right. \\
\left.+\left\{\overline{\nu_{m}} \gamma^{\mu} P_{L} V_{\ell N} N_{m}+\text { H.c. }\right\}\right],
\end{gathered}
$$

where $c_{w}=\cos \theta_{w}$ with $\theta_{w}$ being the weak mixing angle and $W_{\mu}, Z_{\mu}$ are the SM gauge bosons.

The main decay modes of the heavy neutrino are $N \rightarrow \ell W, v_{\ell} Z, v_{\ell} h$. The corresponding partial decay widths [47] are, respectively, given by

$$
\begin{aligned}
& \Gamma(N \longrightarrow \ell W) \\
& \quad=\frac{g^{2}\left|V_{\ell N}\right|^{2}}{64 \pi} \frac{\left(m_{N}^{2}-m_{W}^{2}\right)^{2}\left(m_{N}^{2}+2 m_{W}^{2}\right)}{m_{N}^{3} m_{W}^{2}}, \\
& \Gamma\left(N \longrightarrow v_{\ell} Z\right) \\
& \quad=\frac{g^{2}\left|V_{\ell N}\right|^{2}}{128 \pi c_{w}^{2}} \frac{\left(m_{N}^{2}-m_{Z}^{2}\right)^{2}\left(m_{N}^{2}+2 m_{Z}^{2}\right)}{m_{N}^{3} m_{Z}^{2}}, \\
& \Gamma\left(N \longrightarrow v_{\ell} h\right)=\frac{\left|V_{\ell N}\right|^{2}\left(m_{N}^{2}-m_{h}^{2}\right)^{2}}{32 \pi m_{N}}\left(\frac{1}{v}\right)^{2} .
\end{aligned}
$$

The decay width of heavy neutrino into charged gauge bosons is twice as large as neutral one owing to the two degrees of 


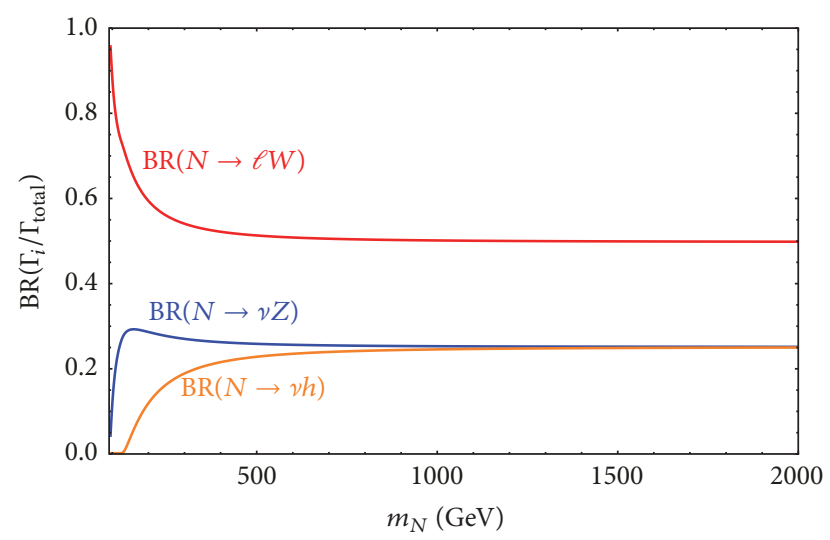

FIGURE 1: Heavy neutrino branching ratios $\left(\mathrm{BR}_{i}\right)$ for different decay modes are shown with respect to its mass $\left(m_{N}\right)$ [51].

freedom $\left(W^{ \pm}\right)$. We plot the branching ratios $\mathrm{BR}_{i}\left(=\Gamma_{i} / \Gamma_{\text {total }}\right)$ of the respective decay modes $\left(\Gamma_{i}\right)$ with respect to the total decay width $\left(\Gamma_{\text {total }}\right)$ of the heavy neutrino into $W, Z$ and Higgs bosons in Figure 1 as a function of the heavy neutrino mass $\left(m_{N}\right)$. Note that for larger values of $m_{N}$ such as $m_{N} \geq$ $1500 \mathrm{GeV}$, the branching ratios can be obtained as

$$
\begin{aligned}
\mathrm{BR} & (N \longrightarrow \ell W): \mathrm{BR}(N \longrightarrow \nu Z): \mathrm{BR}(N \longrightarrow \nu h) \\
\quad & 2: 1: 1 .
\end{aligned}
$$

In this paper we study the RHN production from various initial states such as the quark-quark (qq), quark-gluon (qg), and gluon-gluon (gg) at the $13 \mathrm{TeV}$ LHC and future $100 \mathrm{TeV}$ pp collider. We consider the photon mediated processes as well as the gluon-gluon fusion (ggF), photon-proton interactions, and Vector Boson Fusion (VBF) processes to produce the RHNs. For different RHN masses $\left(m_{N}\right)$; we calculate the cross section of each of the processes normalized by the square of the light-heavy neutrino mixing angles.

This paper is organized as follows. In Section 2 we calculate the production cross sections at the $13 \mathrm{TeV}$ LHC and future $100 \mathrm{TeV}$ pp collider [53] with a variety of initial states. In Section 3 we study the multilepton decay modes of the RHNs at the $13 \mathrm{TeV}$ LHC and future $100 \mathrm{TeV}$ pp collider. For both of the cases we take the luminosity as $3000 \mathrm{fb}^{-1}$. In Section 4 we put the bounds on the mixing angle as function of the RHN mass. Section 5 is dedicated for conclusions.

\section{Production Cross Sections}

The RHNs can be produced at the $13 \mathrm{TeV}$ LHC and future $100 \mathrm{TeV}$ pp collider from various initial states. We consider the combined production of the heavy neutrinos from various initial states including quark-(anti)quark ( $\left.\overline{\mathrm{qq}^{\prime}}\right)$, quarkgluon (qg), and gluon-gluon (gg) interactions. We also study contributions coming from the gluon-gluon fusion (ggF), photon-proton interactions, and Vector Boson Fusion (VBF) processes to produce the RHNs at the LHC and beyond. In this section we consider the RHN productions in association with SM leptons and jets. To obtain the cross sections and

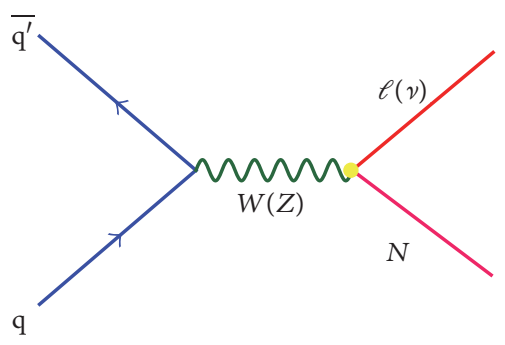

Figure 2: $\mathrm{qq}^{\prime}(\mathrm{q} \overline{\mathrm{q}})$ process to produce $N \ell(N \nu)$ from the CC(NC) interaction.

generate the events we implement our model in MadGraph [54] bundled with PYTHIA [55] and DELPHES [56]. The production modes of the heavy neutrinos in association with leptons and jets are proportional to the square of the mixing angle, $\left|V_{\ell N}\right|^{2}$. In this section we use $\left|V_{\ell N}\right|^{2}=10^{-4}$ to estimate the cross sections. (The EWPD bounds $\left|V_{\ell N}\right|^{2}$ have been discussed in $[20,26,27]$. The universal bound has been considered to be $9 \times 10^{-4}$ which rules out the possibility of the mixing angles above this value. Therefore, we used a value of $\left|V_{\ell N}\right|^{2}<9 \times 10^{-4}$ to calculate the cross sections.)

(1) Charged current interaction is mediated by $W$ :

(i) generate $\mathrm{pp} \rightarrow \mathrm{Nl}$

(ii) add process pp $\rightarrow N \ell j$

(iii) add process pp $\rightarrow N \ell j j$

along with the charge conjugates where $j$ stands for the jets.

In this process the the $s$-channel quark-antiquark pair of different flavors $\left(\mathrm{qq}^{\prime}\right)$ will interact through the $W$ exchange and finally follow (8) to produce the $\mathrm{RHN}(N)$ in association with a lepton $(\ell)$. The corresponding Feynman diagram for $\mathrm{qq}^{\prime} \rightarrow N \ell$ is given in Figure 2.

The contributions from the quark-quark interaction $\left(\mathrm{qq}^{\prime}\right)$ to one-jet $(N \ell j)$ are given in Figure 3 for the $t$-channel process and additional contributions to the one-jet process from the quark-gluon (qg) interaction as shown in Figure 4 form the $s$, $t$-channel processes. In both of the cases $W$ is radiated from the quarks/antiquarks and hence follows the CC to produce $N$ in association with $\ell$.

Two-jet contributions $(N \ell j j)$ coming from the $\mathrm{qq}^{\prime}$ process are shown in Figure 5. In this case, the $s, t$ channel contributions between the quarks are involved to produce $N$ with $\ell$ in association with two jets following the CC interactions at the $N$ production vertex. The quark-gluon (qg) processes contributing in the two-jet case are shown in Figure 6 where the $s, t$ channel contributions between the quarks and gluons are involved to produce $N$ with $\ell$ in association with two jets following the CC interactions at the $N$ production vertex. In addition to that gluon-gluon (gg) 

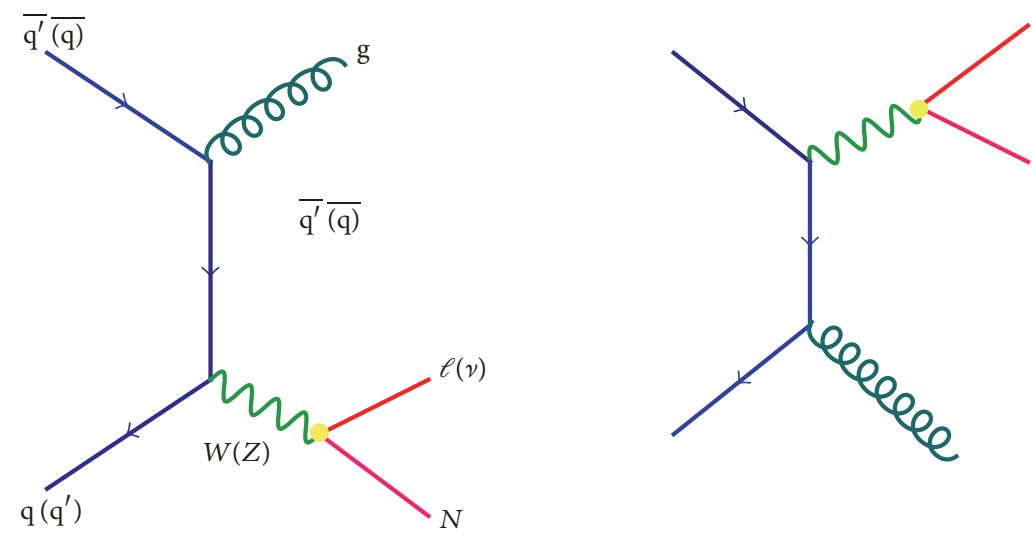

FIGURE 3: 1-jet process to produce $N$ with $\ell(\nu)$ in association with a jet from the CC(NC) interaction from $\mathrm{q} \overline{\mathrm{q}^{\prime}}(\mathrm{q} \overline{\mathrm{q}})$ initial state.
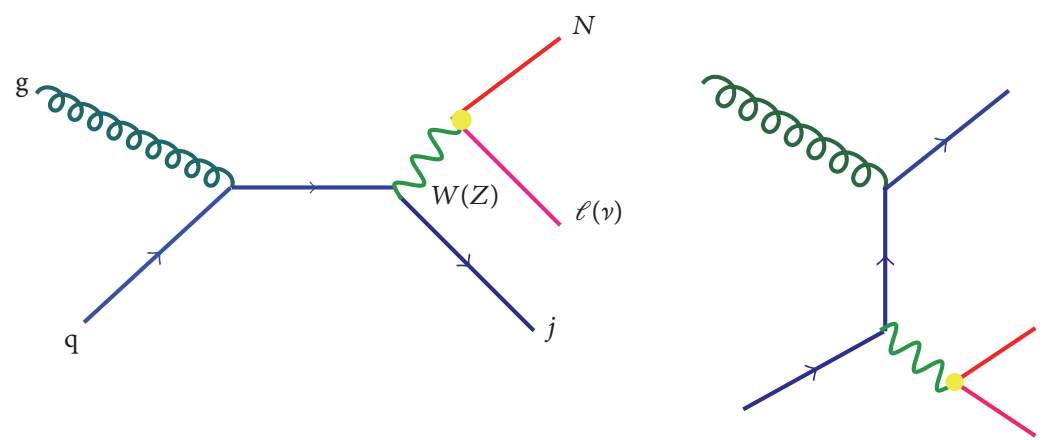

FIGURE 4: 1-jet process to produce $N$ with $\ell(v)$ in association with a jet from the CC(NC) interaction from qg initial state.

processes contributing in $N \ell j j$ are shown in Figure 7 in the $s, t$-channel followed by the $N$ production from the CC interactions with $\ell$ in association with two jets.

We combine all these processes to compute the production cross section of the RHNs through the charged current interaction for N NX final state where $X$ stands for $n$-jet and $n=0,1,2,3$. To produce such processes we use the following trigger cuts:

(a) transverse momentum of the jets, $p_{T}^{j}>30 \mathrm{GeV}$

(b) transverse momentum of the leptons, $p_{T}^{\ell}>$ $10 \mathrm{GeV}$

(c) pseudo-rapidity of the jets, $\left|\eta^{j}\right|<2.5$

(d) pseudo-rapidity of the leptons, $\left|\eta^{\ell}\right|<2.5$,

with the Multi-Leg Matching (MLM) scheme. In the MLM matching scheme one can obtain $n$-parton events by MadEvent generator under the $k_{T}$ clustering algorithm. After the reweight, each final state parton can find the node where it was generated. This $n$ parton shower can be generated $\left(\mu_{g}^{2}\right)$ in the directions of the primary partons so that the initial condition for each parton shower is kept at $\mu_{g}^{2}$. Hence running the $k_{T}$ clustering algorithm one can find all the jets at the resolution scale $\left(\tau_{r}\right)$. If all the jets match with the primary partons then the event is accepted; otherwise it is rejected. To elaborate discussions on this mechanism for the event generators, see [57-69]. In MadGraph the switch ickkw = 1 stands for the MLM scheme in MadGraph [54] using

(i) ickkw $=1$

(ii) xqcut $=p_{T}^{j}$

(iii) $\mathrm{QCUT}=\operatorname{Max}\{$ xqcut $+5 \mathrm{GeV}, 1.2 * \mathrm{xqcut}\}$

using MSTP $(81)=21$. The MSTP switches modify the generation procedure. In this case it switches off or on the Initial State Radiation (ISR), Final State Radiation (FSR), and Multiple Interactions (MI) among the beam jets and fragmentation. It is also used to give the "parton skeleton" of the hard process. $\operatorname{MSTP}(81)=21$ is the master switch in PYTHIA 6.4 which switches on the Multi-Interactions (MI) for the new model at the time of showering and hadronization. The MSTP = 21 is used for the $p_{T}$ ordered shower switching on SHOWER-KT [70-73] in PYTHIA [55]. The trigger cuts are used on the basis of the anomalous multilepton searches done by the CMS $[74,75]$. The use of cone jets or $k_{T}$ jets is decided by whether the parameter xqcut (specifying the minimum $k_{T}$ jet measure between jets, i.e., gluons or quarks (except top quarks) which are connected in Feynman diagrams) in the MadGraph is set to be 0 or not. If xqcut $=0$, cone jets are used, while if xqcut $>0, k_{T}$ jet matching is assumed. 

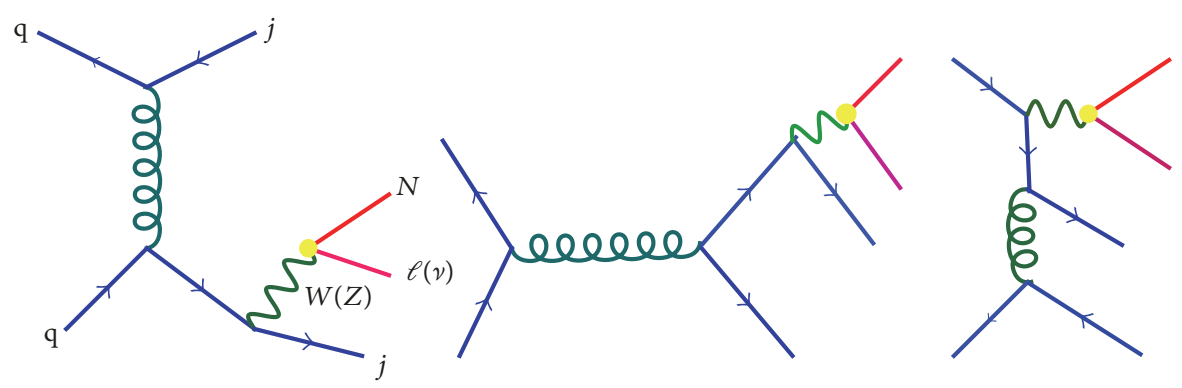

FIGURE 5: 2-jet process to produce $N$ with $\ell(\nu)$ in association with a jet from the CC(NC) interaction from $\mathrm{q} \overline{q^{\prime}}(\mathrm{q} \overline{\mathrm{q}})$ initial state.
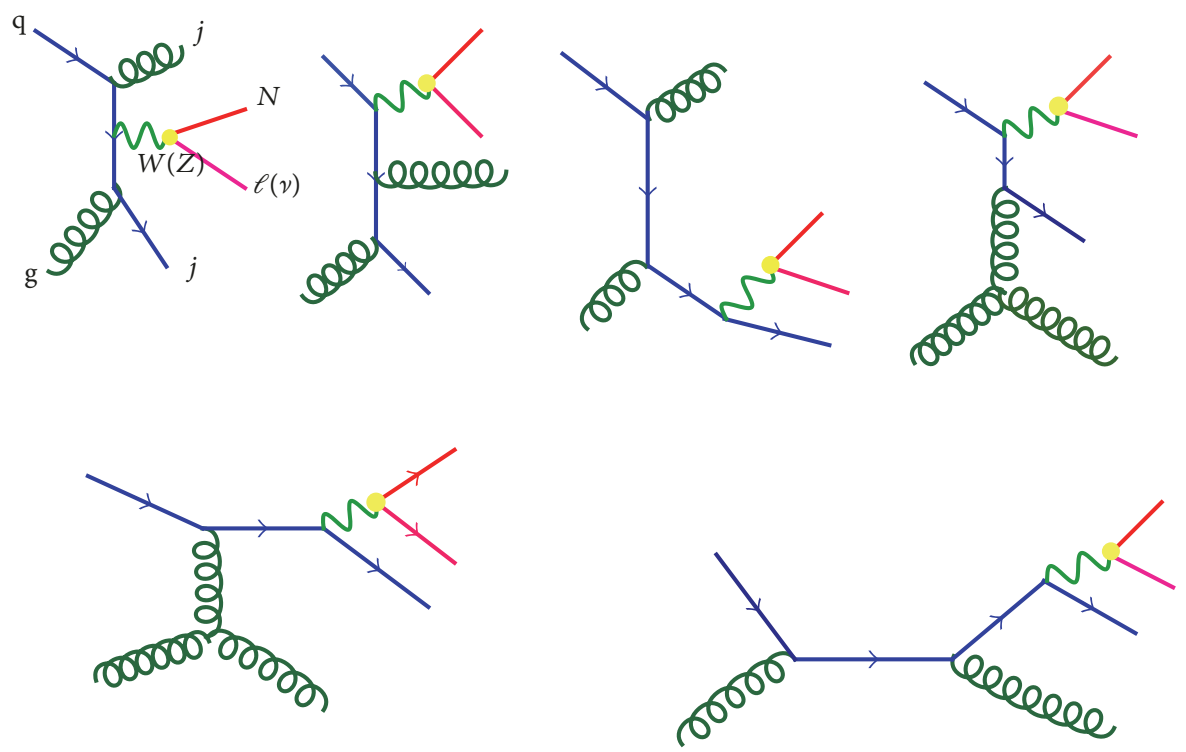

FIGURE 6: 2-jet process to produce $N$ with $\ell(\nu)$ in association with a jet from the CC(NC) interaction from qg initial state.
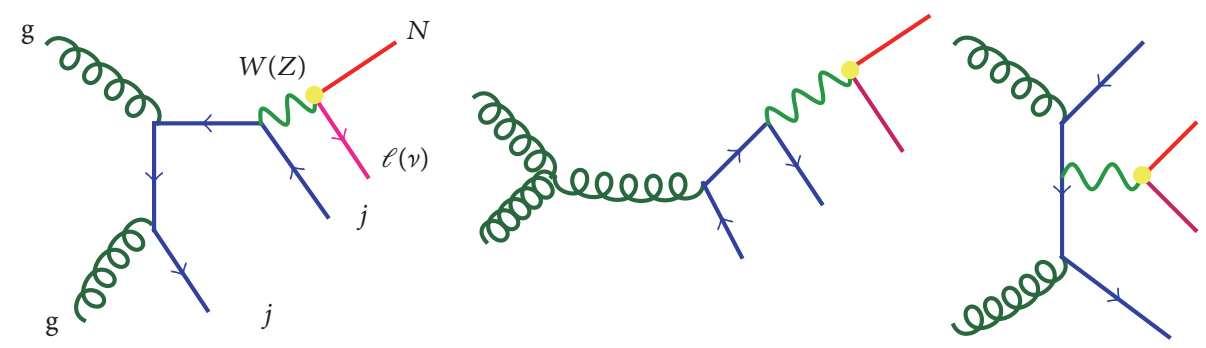

FIgURE 7: 2-jet process to produce $N$ with $\ell(\nu)$ in association with a jet from the CC(NC) interaction from gg initial state.

In this case, transverse momentum of jet $\left(p_{T}^{j}\right)$ and separation between the jets $\left(\Delta R_{j j}\right)$ should be set to zero. For most processes, the generation speed can be improved by setting $\mathrm{p}_{\mathrm{T}}^{\mathrm{j}}=\mathrm{xqcut}$ which is done automatically if the switch auto - ptj - mjj is set to " $T$ " at the time of event generation using MadGraph to control the transverse momentum of the jets $\left(p_{T}^{j}\right)$ and the invariant mass of the jets $\left(m_{j j}\right)$. If some jets should not be restricted this way (as in single top or Vector Boson Fusion (VBF) production, where some jets are not radiated from QCD) in that case the switch auto - $\mathrm{pTj}-\mathrm{mjj}$ should be set to "F" in the MagGraph at the time of event generation.
QCUT is used for the matching with the $k_{T}$ scheme; in this case the jet measure cutoff is used by Pythia. If the value is not given, it will be set to max (xqcut +5 , xqcut $* 1.2$ ), where xqcut is taken from the MadGraph [58, 59]. We use such cuts to keep the analysis collinear safe. The production cross sections are shown in Figures 11 and 12, respectively, as a function of $m_{N}$.

(2) Neutral current interaction is mediated by $Z$ :

(i) generate $\mathrm{pp} \rightarrow \mathrm{N} \nu$

(ii) add process $\mathrm{pp} \rightarrow N \nu j$ 


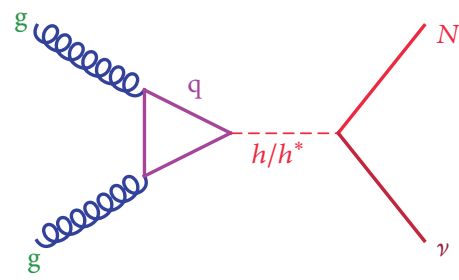

(a)

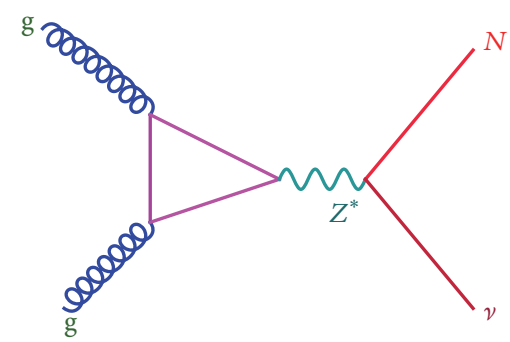

(b)

FIGURE 8: ggF production of the heavy neutrinos from Higgs $(h)$ and $Z$ bosons. $h \rightarrow N v$ can be prompt when $m_{h}>m_{N}$ giving an enhancement to the $N v$ production from the ggF channel. For $m_{h}<m_{N}$, off-shell Higgs $\left(h^{*}\right)$ produces $N v$ final state. Similarly off-shell $Z\left(Z^{*}\right)$ can also produce $N \nu$ final state when $m_{N}>m_{Z}$. For the Higgs mediated case, top quark loop will contribute dominantly whereas for the $Z^{*}$ case all contributions coming from the quarks will be counted according to its coupling with the quarks.

(iii) add process $\mathrm{pp} \rightarrow N \nu j j$

followed by the same production procedure as we discussed in (1). Using quark-antiquark pair of same flavor ( $\mathrm{q} \overline{\mathrm{q}})$ at the $s$-channel by (9) $N$ can be produced in association with a light neutrino $(\nu)$ as shown in Figure 2. The $N v j$ (in Figures 3 and 4 ) and $N v j j$ (in Figures 5, 6, and 7) processes are the same as those which have been discussed in (1). The only difference is the production vertex of $N v$ coming from the NC from (9). Combining these final states we can denote $N v X$ where $X$ stands for $n$-jet with $n=0,1,2,3$. In this case we use the following trigger cuts:

(a) transverse momentum of the jets, $p_{T}^{j}>30 \mathrm{GeV}$

(b) pseudo-rapidity of the jets, $\left|\eta^{j}\right|<2.5$

and the same matching scheme used in (1). Such $p_{T}^{j}$ cuts will keep the analysis collinear safe specially in (1) and (2) to calculate the cross sections using MLM matching [57-61, 66-69] schemes. The production cross sections at the $13 \mathrm{TeV}$ LHC and future $100 \mathrm{TeV}$ pp collider are shown in Figures 11 and 12, respectively, as a function of $m_{N}$.

(3) Gluon-fusion channel (ggF):

In the ggF top loop produces the SM Higgs and Higgs can decay into $N$ in association with $v[45,46,76]$. The corresponding Feynman diagram is given in Figure $8(\mathrm{a})$. There is another complementary production channel of $N$ in association with $v$ which comes from $Z[45,77]$. The corresponding Feynman diagram is given in Figure 8(b). The production process depends on $m_{N}$; one can produce them promptly from the Higgs decay when $m_{N}<m_{h}$, where $m_{h}$ is the Higgs mass and also from the off-shell $Z\left(Z^{*}\right)$. When $m_{N}<$ $m_{h}$, Higgs can decay into RHNs and the partial decay width can be written as

$$
\Gamma_{h}^{\text {new }}=\frac{Y_{D}^{2} m_{H}}{8 \pi}\left(1-\frac{m_{N}^{2}}{m_{h}^{2}}\right)^{2}
$$

This has additive contribution to the total decay width of the SM Higgs boson keeping $Y_{D}$ as a free parameter. Constraints on $Y_{D}$ and $\left|V_{e N}\right|^{2}$ have recently been studied in $[35,45,46]$. The cross section goes down at the $13 \mathrm{TeV}$ LHC for $m_{h}<m_{N}$ due to the off-shell decay. However, at the future $100 \mathrm{TeV}$ pp collider the cross section again rises around $m_{N}=250 \mathrm{GeV}$ due to large contributions coming from the gluon PDFs. In this channel we are testing $m_{N}=100 \mathrm{GeV}-1 \mathrm{TeV}$. The production cross sections are shown in Figures 11 and 12 , respectively, with respect to $m_{N}$. At the future $100 \mathrm{TeV}$, the large gluon PDF starts contributing to the RHN production resulting in a rise in the ggF curve compared to the $13 \mathrm{TeV}$ result.

(4) Photon initiated processes:

The photon (denoted by a in MadGraph) initiated processes also have important contributions in the RHNs production which have been studied in [49, 78-83]. In case of the the photon initiated process, the photon can be radiated from the proton and also from a parton. Those production channels will give additive contributions at the colliders once the RHNs decay into multilepton modes. The corresponding Feynman diagrams for these processes are given in Figure 9. The $N \ell j$ production process is possible as $\gamma \rightarrow W W$ vertex is present which will make the production cross section for $N \ell j$ higher in comparison to that of $N \nu j$ where $\gamma \rightarrow Z Z$ vertex is not present under the SM gauge group. $N \nu j$ will be produced from the $Z \rightarrow N \nu$ vertex under the NC interaction according to (9). For the photon initiated production processes we use

(i) generate pa $\rightarrow \mathrm{N} \ell \mathrm{j}$ QED $=3 \mathrm{QCD}=0$ (QED (Quantum Electrodynamic) processes switches the QED interactions with photon, $W$ and $Z$ bosons, $\mathrm{QED}=3$ means the order of the interaction whereas QCD stands for the Quantum Chromodynamic processes with $\mathrm{QCD}=0$ being the order of the interaction.)

with $p_{T}^{j}>30 \mathrm{GeV}, p_{T}^{\ell}>10 \mathrm{GeV},\left|\eta^{\ell, j}\right|<2.5$. To generate the $N v j$ final state with photon initiated process we use 

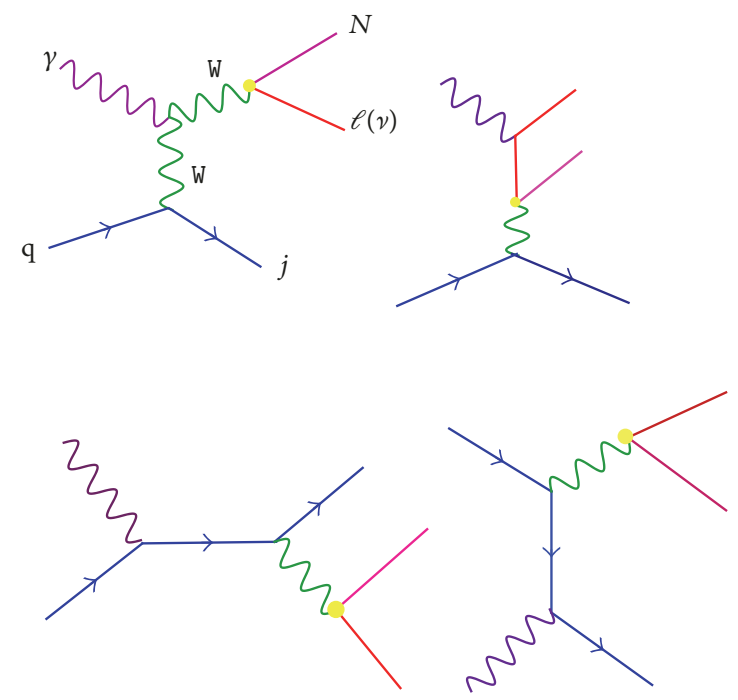

FIgUre 9: The photon initiated $N \ell j$ final state. The $N v j$ final state can also be obtained; however, the contribution will be low as $\gamma \rightarrow$ $\mathrm{ZZ}$ vertex is absent due to the gauge invariance.

(ii) generate $\mathrm{pa} \rightarrow \mathrm{N} v \mathrm{j} \mathrm{QED}=3 \mathrm{QCD}=0$ with $p_{T}^{j}>30 \mathrm{GeV},\left|\eta^{j}\right|<2.5$.

(5) Vector Boson Fusion (VBF) channels:

The VBF processes are shown in Figure 10 where the $N \ell$ and $N v$ are produced with two widely separated jets and the corresponding final states will be $N \ell_{j j}(N v j j)$ depending on the CC (NC) vertex. In the VBF case the $\gamma W W$ and $Z W W$ vertices will come into effect to produce the $N$ in association with $\ell(\nu)$ depending on the CC (NC) of $N-W(N-Z)$. To generate the $N \ell j j(N v j)$ final state from the VBF channels we use

(i) generate $\mathrm{pp} \rightarrow \mathrm{N} \ell \mathrm{j}$ $\mathrm{QED}=4 \mathrm{QCD}=0$ with $p_{T}^{j}>30 \mathrm{GeV}, p_{T}^{\ell}>10 \mathrm{GeV},\left|\eta^{\ell}\right|<2.5$, $\left|\eta^{j_{1}}-\eta^{j_{2}}\right|>4.2, p_{T}^{j_{1} \text {, leading }}=p_{T}^{j_{2} \text {, trailing }}>50 \mathrm{GeV}$, $m_{j j}^{\min }=250 \mathrm{GeV}$.

(ii) generate $\mathrm{pp} \rightarrow \mathrm{N} v \mathrm{jj} \mathrm{QED}=4 \mathrm{QCD}=0$ with $p_{T}^{j}>30 \mathrm{GeV}, p_{T}^{\ell}>10 \mathrm{GeV},\left|\eta^{\ell}\right|<2.5, \mid \eta^{j_{1}}-$ $\eta^{j_{2}} \mid>4.2, p_{T}^{j_{1} \text {,eading }}=p_{T}^{j_{2}}{ }^{\text {trailing }}>50 \mathrm{GeV}, m_{j j}^{\text {min }}=$ $250 \mathrm{GeV}$. The corresponding production cross sections for the $13 \mathrm{TeV}$ LHC and future $100 \mathrm{TeV}$ pp collider are shown in Figures 11 and 12, respectively, with respect to $m_{N}$.

\section{Decay of the RHNs at the LHC}

Implementing our model in the event generator MadGraph, we produce the RHNs and allow it to decay in various multilepton modes. We use kinematic cuts in this adopting from the CMS analysis in [74, 75]. According to our choice we consider two models such as type I Seesaw and Inverse
Seesaw. In type I Seesaw the RHN is Majorana type and in inverse Seesaw the RHN is pseudo-Dirac type. From the Majorana RHN we get LNV same-sign dilepton plus di-jet signal, whereas from the pseudo-Dirac type RHNs we get LNC trilepton plus $\operatorname{MET}\left(E_{T}^{\text {miss }} / \mathbb{E}_{T}\right)$ final state. Considering the leading decay mode of the RHNs $(N \rightarrow W \ell)$ and depending on the choice of the final states, $W$ will decay either leptonically or hadronically. We consider the $13 \mathrm{TeV}$ LHC to study such processes. We consider $m_{N}=100 \mathrm{GeV}$ in this section for different production processes. We use the data files after the hadronization using PYTHIA [55] and detector simulation using DELPHES [56] bundled with MadGrpah [54] and use the MLM matched results as prescribed in the previous section. In this analysis the leading particle is the particle having longest transverse momentum $\left(p_{T}\right)$ distribution whereas the trailing particle is that which has shorter transverse momentum $\left(p_{T}\right)$ distribution. We have plotted the transverse momentum distributions of the leptons $\left(p_{T}^{\ell}\right)$ in Figure 13 and the Missing Transverse Energy $\left(\mathrm{MET} / E_{T}^{\mathrm{miss}} / \mathrm{E}_{T}\right)$ distribution in the Figure 14 from the trilepton plus MET $(3 \ell+$ $\mathrm{MET}+X)$ signal. We notice that the leading lepton transverse momentum, $p_{T}^{\ell}$, for $\ell_{1}$ peaks around $40 \mathrm{GeV}$ whereas the trailing leptons mostly stay between $10 \mathrm{GeV}$ and $40 \mathrm{GeV}$. Looking at the distribution of the leading lepton and the peak of the distribution we suggest that the leading lepton is coming from the leptonic decay of $W$. The MET distribution peaks around $40 \mathrm{GeV}$ which also backs the ides of having the leading lepton of $W$. Therefore, for the trilepton analysis at $m_{N}=100 \mathrm{GeV}$ using $E_{T}^{\text {miss }}<50 \mathrm{GeV}$ will be reasonable choice.

We study the same-sign dilepton plus di-jet $\left(\ell^{ \pm} \ell^{ \pm} j j+\right.$ $X$ ) where $X$ stands for the radiated jets. This signal is an important signature to test the Majorana nature of the RHNs produced from type I Seesaw model. The transverse momentum of the leptons $\left(p_{T}^{\ell}\right)$ and the jets $\left(p_{T}^{j}\right)$ are plotted in Figures 15 and 16, respectively. The invariant mass distribution of the two jets is plotted in Figure 17. The distribution shows a peak around $m_{W}$. The invariant mass distribution of the lepton plus two-jet system has been shown in Figure 18. The figure shows the distributions including $m_{\ell_{1} j j}$ and $m_{\ell_{2} j j}$. Among them $m_{\ell_{1 j}}$ shows a peak around the assumed $m_{N}$ value around $100 \mathrm{GeV}$.

We study the VBF processes to produce the heavy neutrinos and hence study the trilepton plus MET final state. We have used the VBF prescriptions written in the previous section where the jets $j_{1}$ and $j_{2}$ are widely separated. The other two jets are initial state radiations (ISR) which also are not populating the central region. The rapidity distributions of the jets are shown in Figure 19. This is a striking feature of the VBF process. The leading lepton coming out of the $W$ decay after the $W$ boson is produced from the $N \rightarrow W \ell$ decay. The leading leptons are also slightly away from the central region. We use a lepton pseudo-rapidity cut, $\left|\eta^{\ell}\right|<$ 2.5. The rapidity distributions of the leptons are shown in Figure 20.

Another important contribution is coming from the $N v j j+X$ process from the NC interaction between the RHNs and $Z$ bosons. Here $X$ are the radiated jets. The final state 

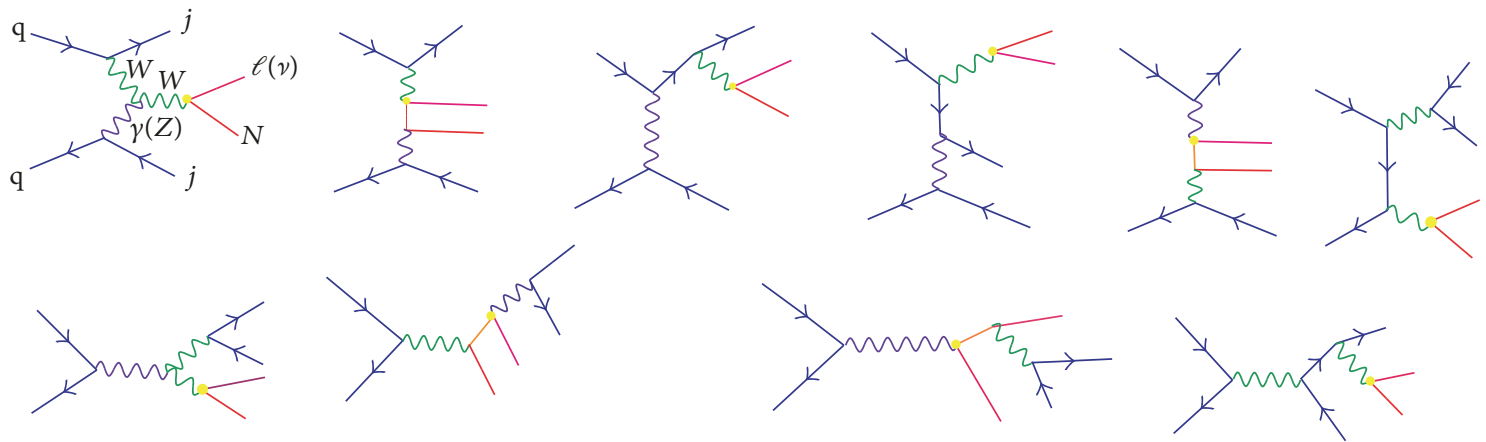

Figure 10: VBF production of the $N \ell j j$ final state from the $W \rightarrow N \ell$ vertex. The $N \nu j j$ finals state can also be obtained using the $Z \rightarrow N \nu$ vertex. The two jets can be obtained at the forward-backward regions.

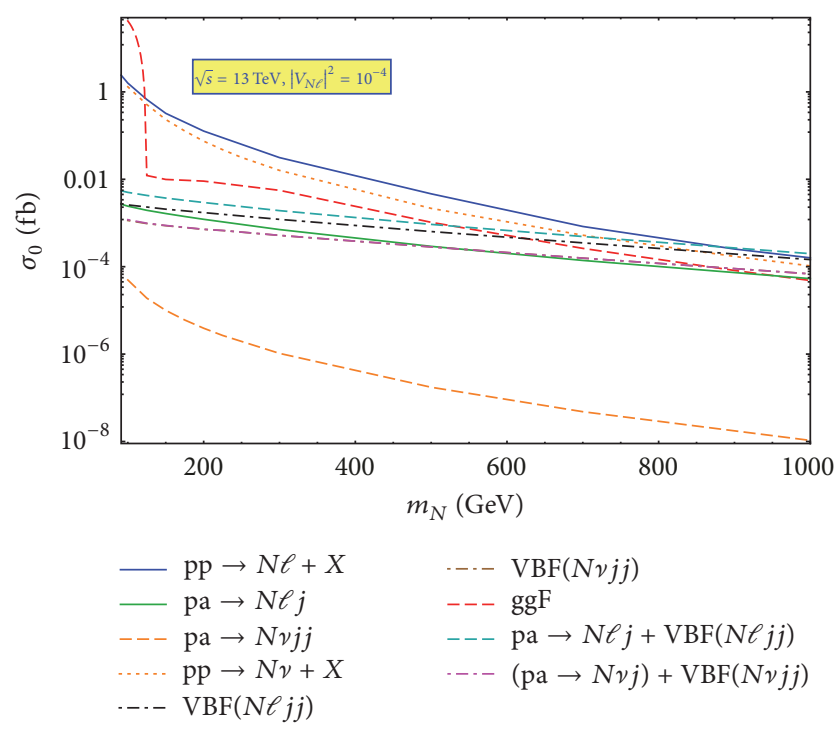

FIGURE 11: Production cross sections of the RHNs in association with leptons from different modes at the $13 \mathrm{TeV}$ LHC with $\left|V_{\ell N}\right|^{2}=10^{-4}$. "a" stands for photon $(\gamma)$ as it is defined by MadGraph.

consists of a single lepton, two jets, and MET. The produced jets can be used to reconstruct the $W$ boson. The distribution shows a peak around the $W$ mass. Similarly from the RHN decay we get $N \rightarrow \ell j j$ and the invariant mass distribution of the $\ell j j$ system can show a peak around the RHN mass $\left(m_{N}\right)$. The invariant mass distribution of $m_{j j}$ is shown in Figure 21 and that of $m_{\ell j j}$ is shown in Figure 22 .

We produced the RHNs from the ggF process where the top loop becomes dominant to produce the Higgs which can promptly decay into the RHN when $m_{N}<m_{H}$. In our case we consider the $m_{N}=100 \mathrm{GeV}$ and the Higgs will decay promptly into $N \nu$ and the RHN will decay into $W$ followed by its hadronic decay. The invariant mass distribution of the two-jet system $\left(m_{j j}\right)$ is shown in Figure 23 whereas that of the lepton and two-jet system are shown in Figure 24. The $m_{j j}$ distribution peaks around the $W$ mass whereas the $m_{\ell j j}$ distribution peaks around the RHN mass. Detailed results regarding the Higgs decaying into RHNs can be found in $[35,45,46]$ with pervious and updated limits.

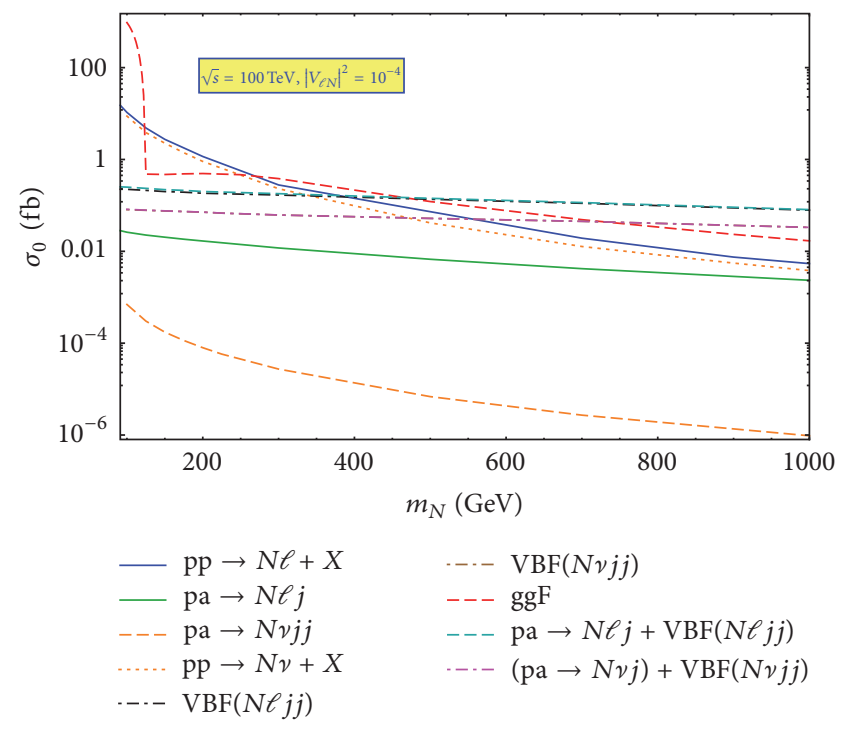

FIGURE 12: Production cross sections of the RHNs in association with leptons from different modes at the future $100 \mathrm{TeV}$ pp collider with $\left|V_{\ell N}\right|^{2}=10^{-4}$. "a" stands for photon $(\gamma)$ as it is defined by MadGraph.

\section{Bounds on the Mixing Angle}

For $m_{N}<M_{Z}$, the RHN can be produced from the onshell $Z$-decay through the NC interaction in association with missing energy; however, if $m_{N}>M_{Z}$ off-shell production will take place with the same final state. The heavy neutrino can decay according to the CC and NC interactions. Different production processes, different decay modes of the heavy neutrinos, and various phenomenologies have been discussed in $[51,52,82,84-94]$. RHN production from various initial states and scale dependent production cross sections at the Leading Order (LO) and Next-to-Leading-Order QCD (NLO QCD) of $N v$ have been studied at the $14 \mathrm{TeV}$ LHC and future $100 \mathrm{TeV}$ pp collider [51, 52]. The L3 collaboration [21] has performed a search on such heavy neutrinos directly from the LEP data and found a limit on $\mathscr{B}(Z \rightarrow v N)<3 \times 10^{-5}$ at the $95 \% \mathrm{CL}$ for the mass range up to $93 \mathrm{GeV}$. The exclusion limits from L3 are given in Figure 25 where the red solid line stands for the limits obtained from electron (L3-e) and the 


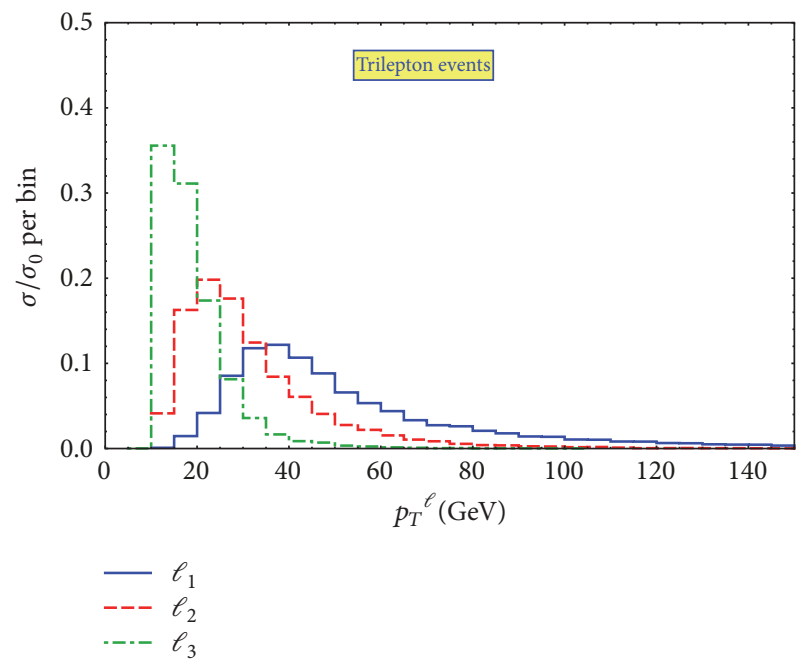

FIgURE 13: Transverse momenta distributions for the three leptons from the $3 \ell+$ MET final state from pp $\rightarrow N \ell X, N \rightarrow \ell W, W \rightarrow \ell v$ at the $13 \mathrm{TeV}$ LHC.

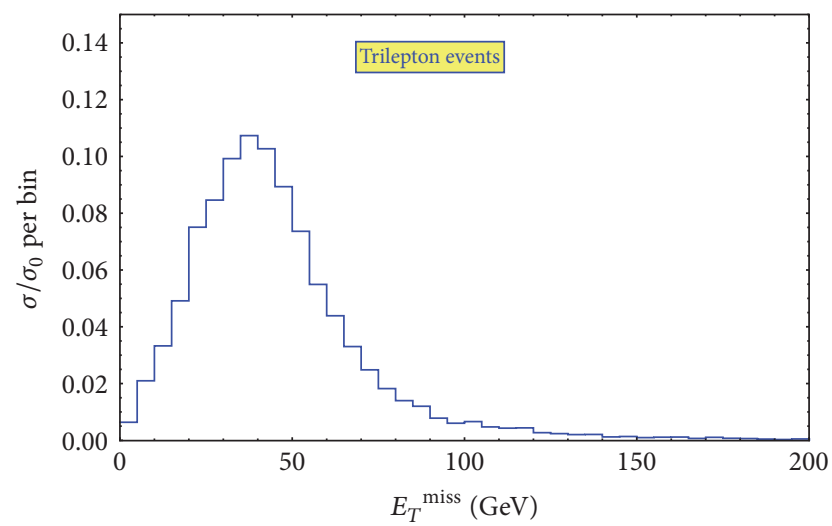

FIGURE 14: MET distribution from the $3 \ell+$ MET final state from $\mathrm{pp} \rightarrow N \ell X, N \rightarrow \ell W, W \rightarrow \ell \nu$ at the $13 \mathrm{TeV}$ LHC.

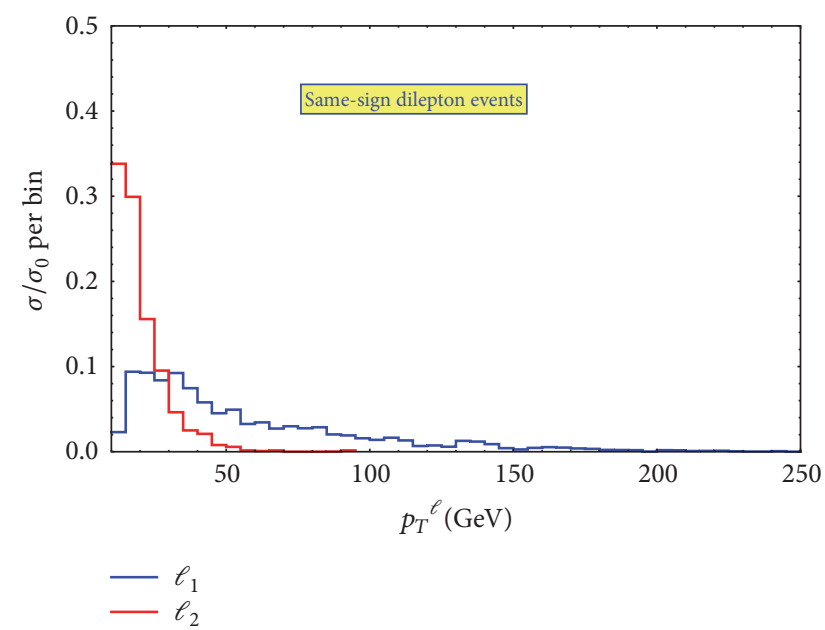

FIGURE 15: Transverse momentum distributions of leptons $\left(p_{T}^{\ell}\right)$ from the same-sign dilepton plus di-jet final state events.

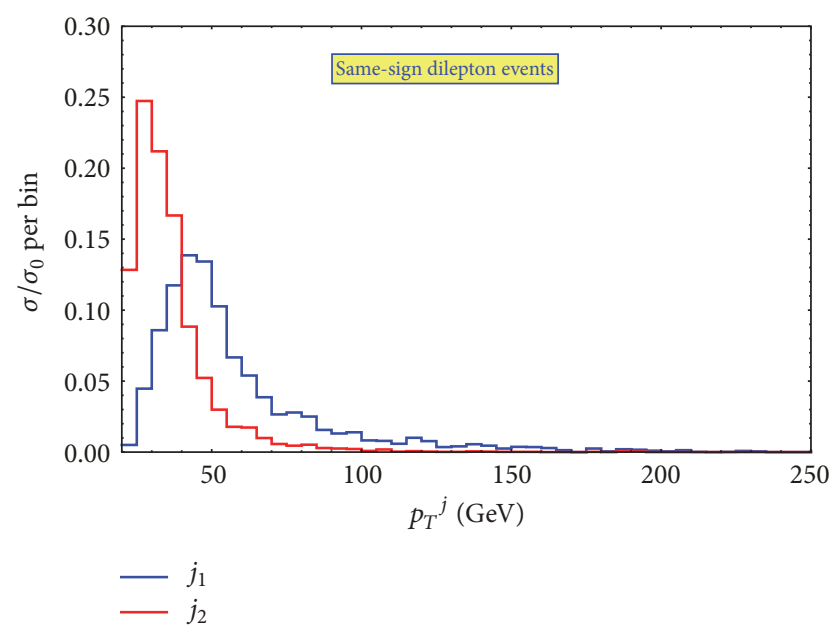

FIGURE 16: Transverse momentum distributions of the jets $\left(p_{T}^{j}\right)$ from the same-sign dilepton plus di-jet final state.

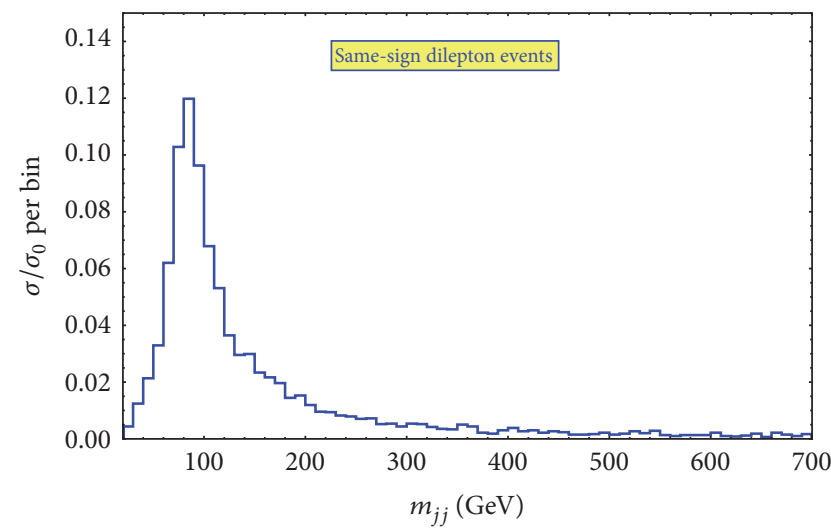

FIGURE 17: The invariant mass distributions of two-jet system $\left(m_{j j}\right)$ from the same-sign dilepton plus di-jet final state showing a peak around the $W$ mass $\left(m_{W}\right)$.

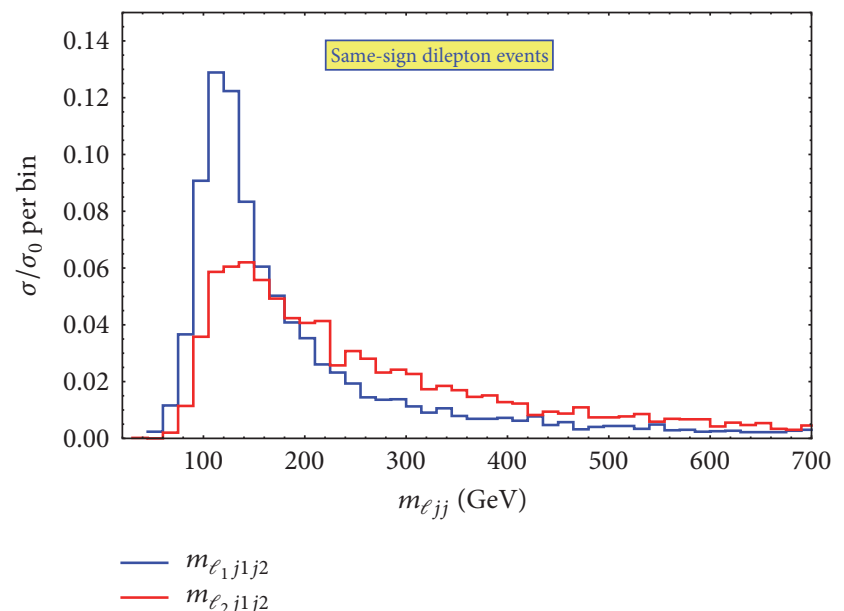

FIGURE 18: The invariant mass distributions of one lepton plus twojet system $\left(m_{l j j}\right)$ showing a peak around $m_{N} \sim 100 \mathrm{GeV}$ (blue) from the same-sign dilepton plus di-jet signal. 


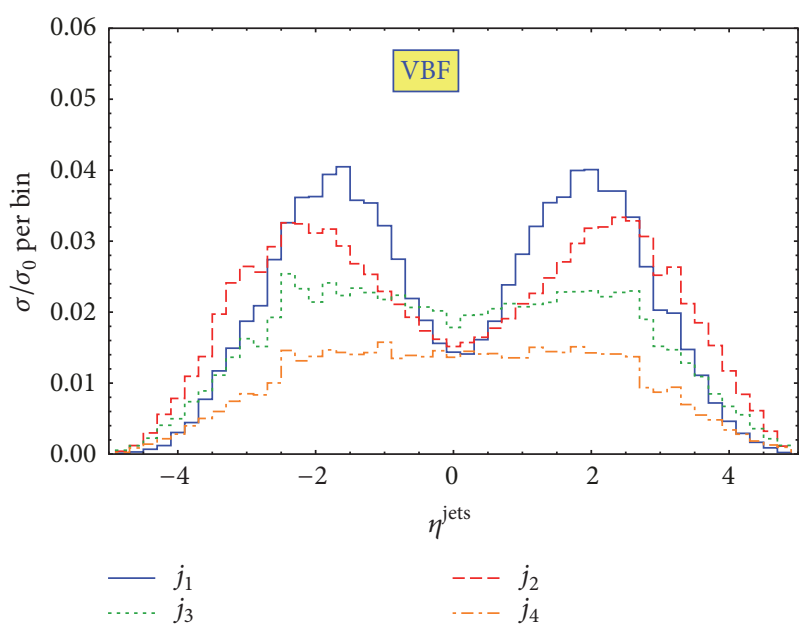

FIGURE 19: Rapidity distributions of the associated jets in the trilepton mode from VBF process.

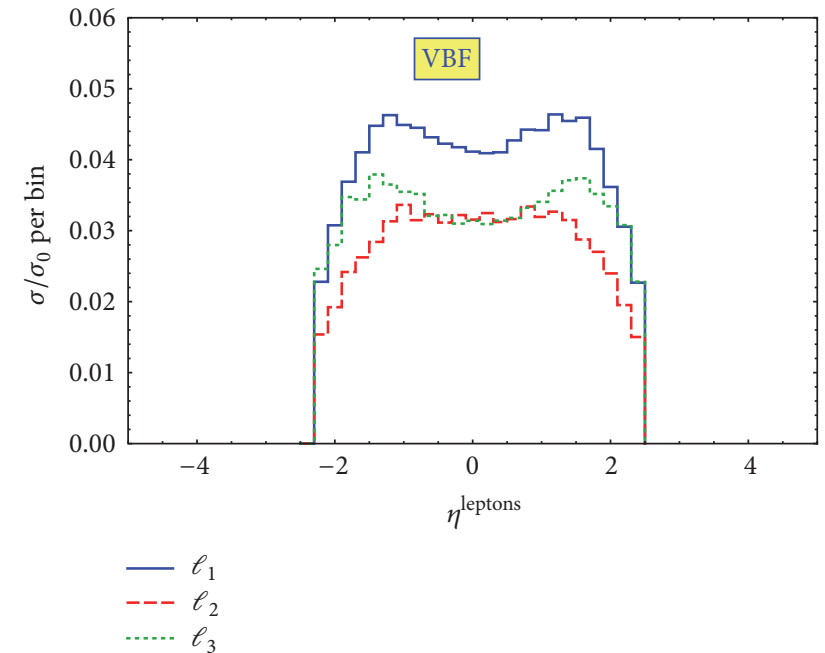

FIGURE 20: Rapidity distributions of the the leptons in the trilepton mode from VBF process.

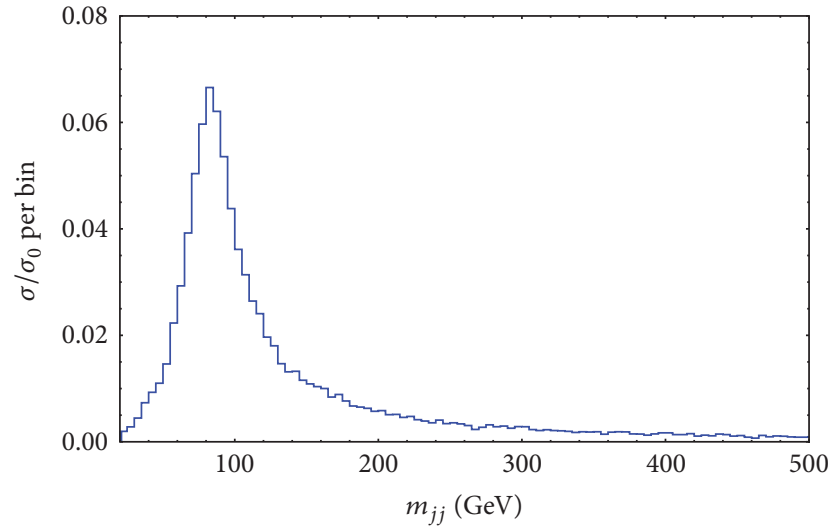

FIGURE 21: Invariant mass distribution of the two-jet system $\left(m_{j j}\right)$ from the $\ell+\mathrm{MET}+2 j+X$ final state from the $\mathrm{pp} \rightarrow N v X$ process. The two-jet system shows a peak around the $W$ mass $\left(m_{W}\right)$.

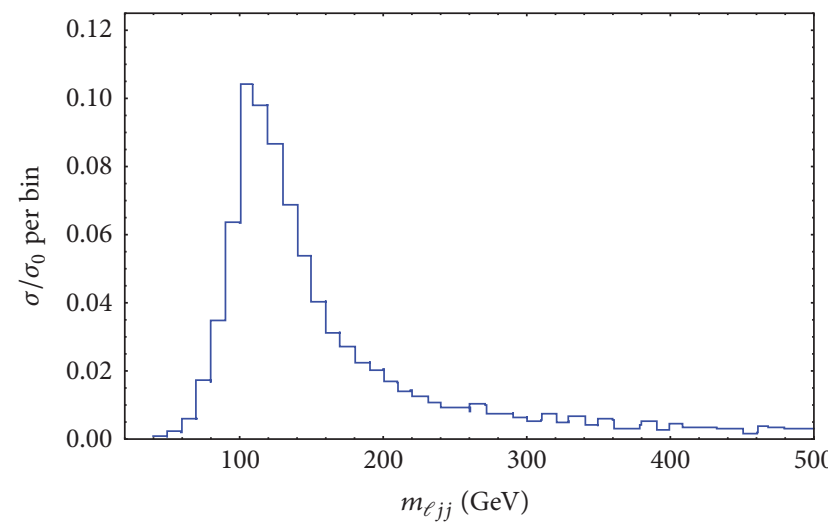

FIGURE 22: Invariant mass distribution of the lepton plus two-jet systems $\left(m_{\ell j j}\right)$ from the $\ell+$ MET $+2 j+X$ final state from the pp $\rightarrow N v X$ process. The lepton plus two-jet system shows a peak around $m_{N} \sim 100 \mathrm{GeV}$.

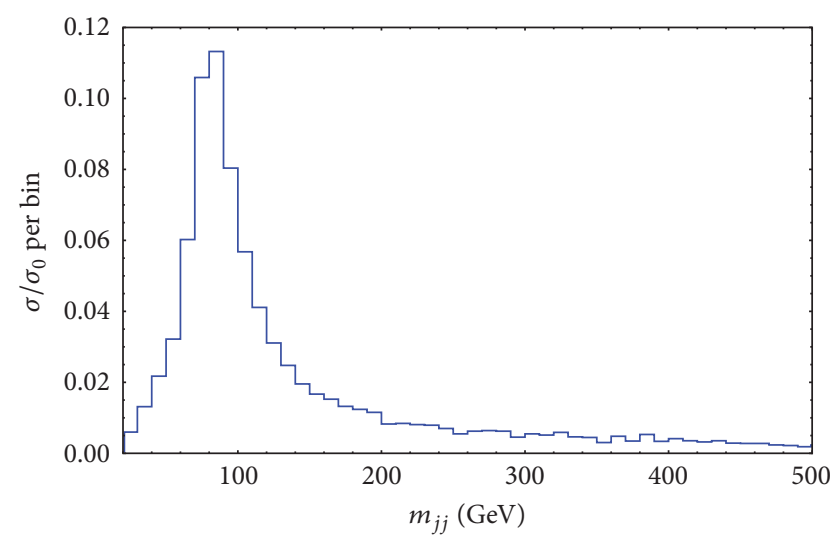

FIgURE 23: Invariant mass distribution of the two-jet system $\left(m_{j j}\right)$ from the $\ell+\mathrm{MET}+2 j$ final state coming from ggF process. The two-jet system shows a peak around the $W$ mass $\left(m_{W}\right)$.

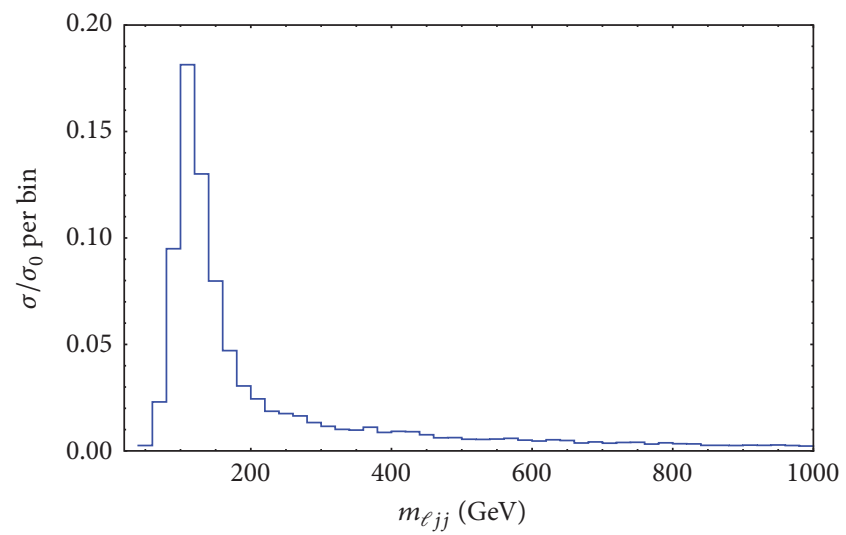

FIgURE 24: Invariant mass distribution of the lepton plus two-jet systems $\left(m_{\ell j j}\right)$ from the $\ell+\mathrm{MET}+2 j$ final state coming from ggF process. The lepton plus two-jet system shows a peak around the RHN mass $\left(m_{N}\right)$ at the $13 \mathrm{TeV}$ LHC. 


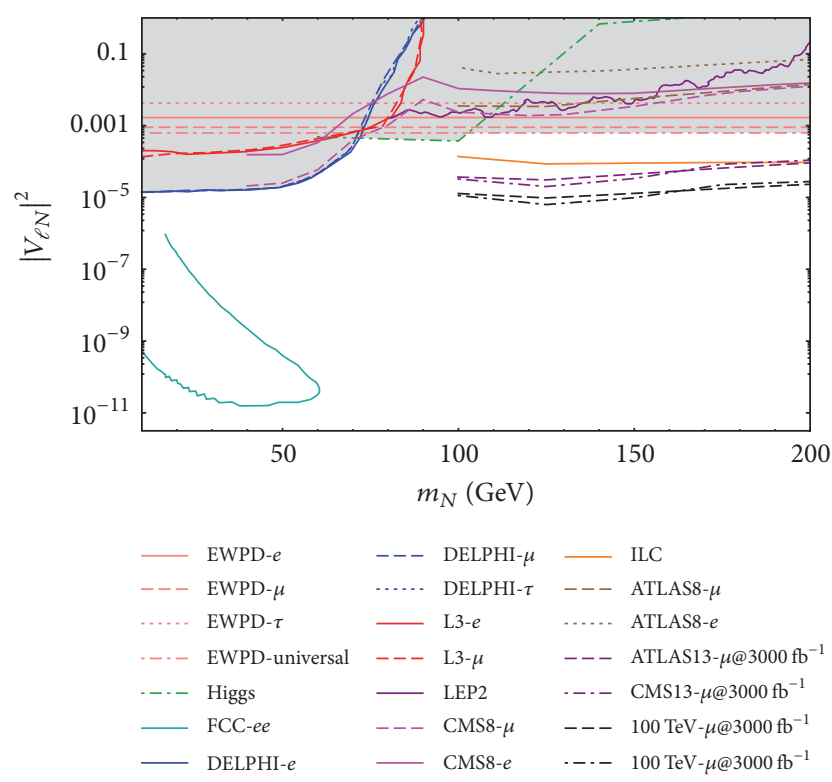

FIGURE 25: Bounds on the square of the mixing angle from the same-sign dilepton plus di-jet final state as a function of the heavy neutrino mass $\left(m_{N}\right)$. The shaded region is ruled out by DELPHI, EWPD, and Higgs data. Prospective bounds from the Higgs data had been calculated in [45] for the LHC at the high luminosity $\left(3000 \mathrm{fb}^{-1}\right)$ and future $100 \mathrm{TeV}$ pp collider.

red dashed line stands for the exclusion limits coming from $\mu$ $(\mathrm{L} 3-\mu)$. The corresponding exclusion limits on $\left|V_{(\ell=e) N}\right|$ at the 95\% CL $[22,23]$ have been drawn from the LEP2 data which have been denoted by the dark magenta line. In this analysis they searched for $80 \mathrm{GeV} \leq M_{N} \leq 205 \mathrm{GeV}$ with a center of mass energy between $130 \mathrm{GeV}$ and $208 \mathrm{GeV}$ [23].

The DELPHI collaboration [24] had also performed the same search from the LEP-I data which set an upper limit for the branching ratio $\mathscr{B}(Z \rightarrow N v)$ about $1.3 \times 10^{-6}$ at $95 \% \mathrm{CL}$ for $3.5 \mathrm{GeV} \leq m_{N} \leq 50 \mathrm{GeV}$. Outside this range the limit starts to become weak with the increase in $m_{N}$. Here it has been considered that $N \rightarrow W \ell$ and $N \rightarrow Z v$ decay after the production of the heavy neutrino was produced. The exclusion limits for $\ell=e, \mu, \tau$ are depicted by the blue solid, dashed, and dotted lines in Figure 25.

The search of the sterile neutrinos can be made at high energy lepton colliders with a very high luminosity such as Future Circular Collider (FCC) for the Seesaw model. A design of such collider has been launched recently where nearly $100 \mathrm{~km}$ tunnel will be used to study high luminosity $e^{+} e^{-}$collision (FCC-ee) with a center-of-mass energy around $90 \mathrm{GeV}$ to $350 \mathrm{GeV}$ [25]. According to this report, a sensitivity down to $\left|V_{\ell N}\right|^{2} \sim 10^{-11}$ could be achieved from a range of the heavy neutrino mass, $10 \mathrm{GeV} \leq m_{N} \leq 80 \mathrm{GeV}$. The darker cyan-solid line in Figure 25 shows the prospective search reaches by the FCC-ee. A sensitivity down to a mixing of $\left|V_{\ell N}\right|^{2} \sim 10^{-12}$ can be obtained in FCC-ee [25], covering a large phase space for $m_{N}$ from $10 \mathrm{GeV}$ to $80 \mathrm{GeV}$.

The heavy neutrinos can participate in many electroweak (EW) precision tests due to the active-sterile couplings. For comparison, we also show the 95\% CL indirect upper limit on the mixing angle, $\left|V_{\ell N}\right|<0.030,0.041$ and 0.065 for $\ell=$ $\mu, e, \tau$ respectively, derived from a global fit to the electroweak precision data (EWPD), which is independent of $m_{N}$ for $m_{N}>M_{Z}$, as shown by the horizontal pink dash, solid, and dolled lines, respectively, in Figure 25. The bounds from the EWPD have been studied in [20, 26, 27]. For the mass range, $m_{N}<M_{Z}$, it is shown in [83] that the exclusion limit on the mixing angle remains almost unaltered; however, it varies drastically at the vicinity of $m_{N}=1 \mathrm{GeV}$. For the flavor universal case the bound on the mixing angle is given as $\left|V_{\ell N}\right|^{2}=0.025$ from [20] which has been depicted in Figure 25 with a pink dot-dashed line.

The CMS limits from the $8 \mathrm{TeV}$ LHC are represented by the Plink lines. The dashed Pink line represents $\mu$ and solid pink line represents $e$. For both of the flavors CMS has tested the mass range, $40 \mathrm{GeV} \leq m_{N} \leq 200 \mathrm{GeV}$. For electron it has probed down to $10^{-4}$ and for muon it has probed down to $10^{-5}$ for $\left|V_{e N}\right|^{2}$ and $\left|V_{\mu N}\right|^{2}$, respectively. The ATLAS bounds from the $8 \mathrm{TeV}$ LHC are weaker than the CMS bounds. The ATLAS [36] bounds are represented by the brown dashed lines for $\mu\left(\left|V_{\mu N}\right|^{2}\right)$ and brown dotted line represents the bounds from $e\left(\left|V_{e N}\right|^{2}\right)$ in Figure 25.

The relevant existing upper limits at the $95 \%$ CL are also shown to compare with the experimental bounds using the LHC Higgs boson data in [35, 48] using [95-99]. The darker green dot-dashed line named Higgs boson shows the relevant bounds on the mixing angle. In this analysis we will compare our results taking this line as one of the references. We have noticed that $\left|V_{\ell N}\right|^{2}$ can be as low as $4.86 \times 10^{-4}$ while $m_{N}=$ $60 \mathrm{GeV}$ and the bound becomes stronger at $m_{N}=100 \mathrm{GeV}$ as $3.73 \times 10^{-4}$. When $m_{N}>100 \mathrm{GeV}$, the bounds on $\left|V_{\ell N}\right|^{2}$ become weaker.

4.1. Same-Sign Dilepton Plus Di-Jet Signal. For simplicity we consider the case that only one generation of the heavy neutrino is light and accessible to the LHC which couples to only the second generation of the lepton flavor. To generate the events in the MadGraph we use the CTEQ6L1 PDF [100] with xqcut $=p_{T}^{j}=20 \mathrm{GeV}$ and QCUT $=25 \mathrm{GeV}$. We calculate the cross sections for the combined processes $N \ell X, N \rightarrow \ell j j$ as functions of $m_{N}$ from various initial states as described in Figures 11 and 12. Comparing our generated events with the recent ATLAS results [36] at the $8 \mathrm{TeV}$ LHC with the luminosity $20.3 \mathrm{fb}^{-1}$, we obtain an upper limit on the mixing angles between the Majorana type heavy neutrino and the SM leptons as a function of $m_{N}$. In the ATLAS analysis the upper bound of the production cross section ( $\sigma^{\text {ATLAS/CMS }}$ ) is obtained for the final state with the same-sign di-muon plus di-jet as a function of $m_{N}$. For $\sigma^{\text {ATLAS }}$ results we use the cross sections given in [36] and for $\sigma^{\mathrm{CMS}}$ we use [101]. Implementing our model in MadGraph we generate the signal event and compare the experimental cross sections from ATLAS and CMS using

$$
\begin{aligned}
& \left|V_{\mu N}\right|^{2} \\
& \lesssim \frac{\sigma^{\text {ATLAS/CMS }}}{(\mathrm{pp} \rightarrow N \ell X, N \rightarrow \ell j j)+\text { All same sign dilepton modes }} .
\end{aligned}
$$


Our upper bounds for the $13 \mathrm{TeV}$ LHC with $3000 \mathrm{fb}^{-1}$ luminosity on the mixing angles are shown in Figure 25 along with the exclusion limits obtained from the different experimental results shaded in gray as described in the previous subsection. We can see that a significant improvement on the bounds can be made adding the jets and various initial states as described in this paper. Applying the ATLAS bound at $8 \mathrm{TeV}$ with $20.3 \mathrm{fb}^{-1}$ luminosity [36], we put a prospective upper bound on the mixing angles at the $13 \mathrm{TeV}$ LHC with $3000 \mathrm{fb}^{-1}$ luminosity. Recently the CMS has performed the same-sign dilepton plus di-jet search [101]. Using this result and adopting the same procedure for the ATLAS result we calculate the upper bound on the mixing angles at the $13 \mathrm{TeV}$ LHC with $3000 \mathrm{fb}^{-1}$ luminosity using (13). The results are shown in Figure 25.

A significant improvement for the upper bound on the mixing angle is obtained by combining all the processes with jets and various initial states as described in this paper. We can see that, at $91.2 \mathrm{GeV} \leq m_{N} \leq 200 \mathrm{GeV}$, the bounds obtained using the ATLAS (ATLAS13- $\mu @ 3000 \mathrm{fb}^{-1}$ ) analysis is better than the recent exclusions limits shaded in gray. Using the $8 \mathrm{TeV}$ CMS result [101] we obtain prospective bound at the $13 \mathrm{TeV}$ LHC for the luminosity $3000 \mathrm{fb}^{-1}$ (CMS13$\left.\mu @ 3000 \mathrm{fb}^{-1}\right)$ in Figure 25. The results are also shown in Figure 25. In this analysis we have only chosen the $\mu$ flavor. The purple lines represent the $13 \mathrm{TeV}$ LHC and black lines represent the future $100 \mathrm{TeV}$ pp collider. The corresponding improved bounds at the future $100 \mathrm{TeV}$ pp collider with $3000 \mathrm{fb}^{-1}$ luminosity are shown in Figure 25 with the black dashed and dot-dashed lines corresponding to the ATLAS and CMS data marked as $\left(100 \mathrm{TeV}-\mu @ 3000 \mathrm{fb}^{-1}\right)$, respectively, in Figure 25. The prospective bounds calculated from (13) at the $13 \mathrm{TeV}$ LHC are represented by the purple and those for the future $100 \mathrm{TeV}$ are represented by black lines.

We find that, at the HL-LHC, using the same-sign dimuon and di-jet final state LHC can probe the squared of the mixing angle down to $1.95 \times 10^{-5}$ for the $\mu$ flavor whereas the future $100 \mathrm{TeV}$ pp collider can probe the square of the mixing angle down to $5.83 \times 10^{-6}$ for the $\mu$ flavor.

4.2. Trilepton Signal. In this analysis we consider two cases. One is the Flavor Diagonal (FD) case, where we introduce three generations of the degenerate heavy neutrinos and each generation couples with the single, corresponding lepton flavor. The other one is the Single Flavor (SF) case where only one heavy neutrino is light and accessible to the LHC which couples to only the first or second generation of the lepton flavor using the CTEQ6L PDF [100]. In this analysis we consider $N \ell X$ final state followed by $N \rightarrow \ell \ell v_{\ell}$.

We generate the combined parton level events using MadGraph and then gradually hadronize and perform detector simulations with xqcut $=p_{T}^{j}=30 \mathrm{GeV}$ and QCUT $=$ $36 \mathrm{GeV}$ for the hadronization. In our analysis we use the matched cross section after the detector level analysis. After the signal events are generated we adopt the following basic criteria, used in the CMS trilepton analysis [74, 75]:

(i) The transverse momentum of each lepton: $p_{T}^{\ell}>$ $10 \mathrm{GeV}$ (ii) The transverse momentum of at least one lepton: $p_{T}^{\ell, \text { leading }}>20 \mathrm{GeV}$

(iii) The jet transverse momentum: $p_{T}^{j}>30 \mathrm{GeV}$

(iv) The pseudo-rapidity of leptons: $\left|\eta^{\ell}\right|<2.4$ and of jets: $\left|\eta^{j}\right|<2.5$

(v) The lepton-lepton separation: $\Delta R_{\ell \ell}>0.1$ and the lepton-jet separation: $\Delta R_{\ell j}>0.3$

(vi) The invariant mass of each OSSF (opposite-sign same flavor) lepton pair: $m_{\ell^{+} \ell^{-}}<75 \mathrm{GeV}$ or $>105 \mathrm{GeV}$ to avoid the on $Z$ region which was excluded from the CMS search. Events with $m_{\ell^{+} \ell^{-}}<12 \mathrm{GeV}$ are rejected to eliminate background from low-mass Drell-Yan processes and hadronic decays

(vii) The scalar sum of the jet transverse momenta: $H_{T}<$ $200 \mathrm{GeV}$

(viii) The missing transverse energy: $\mathbb{E}_{T}<50 \mathrm{GeV}$

The additional trilepton contributions come from $N \rightarrow$ $Z v, h v$, followed by the $Z, h$ decaying into a pair of OSSF leptons. However, the $Z$ contribution is rejected after the implementation of the invariant mass cut for the OSSF leptons to suppress the SM background and the $h$ contribution is suppressed due to very small Yukawa couplings of electrons and muons. Using different values of $E_{T}$ and $H_{T}$, the CMS analysis provides different number of observed events and the corresponding SM background expectations. For our analysis the set of cuts listed above are the most efficient ones as implemented by the CMS analysis $[74,75]$. To derive the limits on $\left|V_{\ell N}\right|^{2}$, we calculate the signal cross section normalized by the square of the mixing angle as a function of the heavy neutrino mass $m_{N}$ for both SF and FD cases, by imposing the CMS selection criteria listed above. The corresponding number of signal events passing all the cuts is then compared with the observed number of events at the $19.5 \mathrm{fb}^{-1}$ luminosity $[74,75]$. For the selection criteria listed above, the CMS experiment observed the following:

(a) 510 events with the SM background expectation of $560 \pm 87$ events for $m_{\ell^{+} \ell^{-}}<75 \mathrm{GeV}$ which is called below the $Z$-pole

(b) 178 events with the SM background expectation of $200 \pm 35$ events for $m_{\ell^{+} \ell^{-}}>105 \mathrm{GeV}$ which is called above the $Z$-pole

In case (a) we have an upper limit of 37 signal events, while case (b) leads to an upper limit of 13 signal events. Using the limits obtained in case (b), we can set an upper bound on $\left|V_{\ell N}\right|^{2}$ for a given value of $m_{N}$. In this analysis we use above the $Z$-pole situation with $3000 \mathrm{fb}^{-1}$ luminosity for the $13 \mathrm{TeV}$ LHC and future $100 \mathrm{TeV}$ pp collider. (Exactly the same procedure can be adopted for the situation with $m_{\ell^{+} \ell^{-}}<$ $75 \mathrm{GeV}$.)

The mixing-squared versus $m_{N}$ contours shown in Figure 26 are allowed by the EWPD within the range $91.2 \mathrm{GeV}$ $\leq m_{N} \leq 163 \mathrm{GeV}$ for the SF case at the $13 \mathrm{TeV}$ LHC at $3000 \mathrm{fb}^{-1}$ luminosity. At the same time we analyze the FD case through which one can probe the range of $91.2 \mathrm{GeV} \leq m_{N} \leq$ 


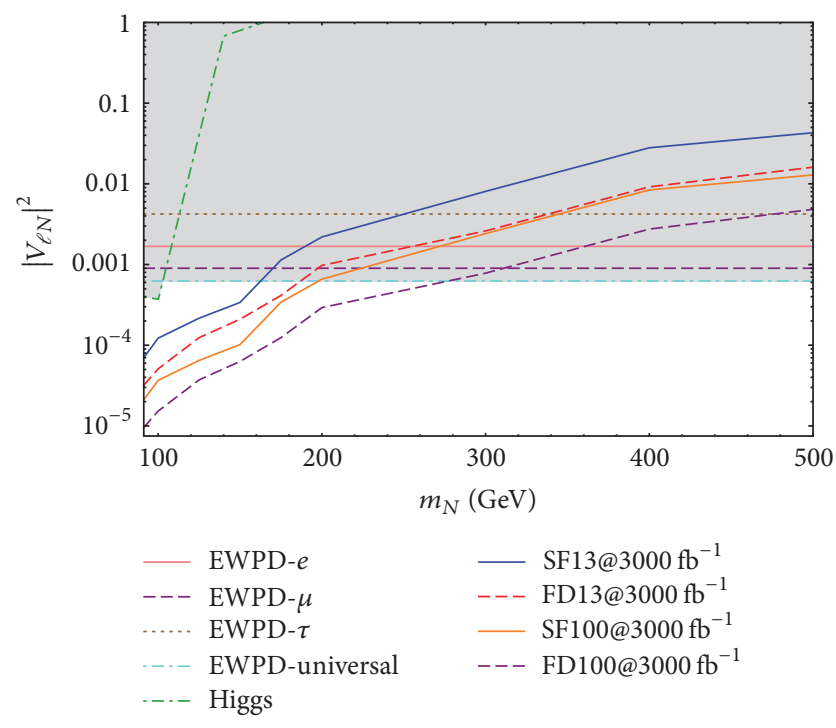

FIGURE 26: Bounds on the square of the mixing angle from the trilepton plus MET final state as a function of the heavy neutrino mass $\left(m_{N}\right)$. The shaded region is ruled out by the EWPD and Higgs data. Prospective bounds from the Higgs data had been calculated in [45] for the LHC at the high luminosity $\left(3000 \mathrm{fb}^{-1}\right)$ and future $100 \mathrm{TeV}$ pp collider.

$195 \mathrm{GeV}$. The limits for the FD case are roughly twice stronger than the SF case. However, at the future $100 \mathrm{TeV}$ pp collider with $3000 \mathrm{fb}^{-1}$ luminosity the upper bounds on $m_{N}$ go up to $200 \mathrm{GeV}$ and $275 \mathrm{GeV}$ for the SF and FD cases, respectively, keeping lower mass bound at the $91.2 \mathrm{GeV}$. As the coupling between the Higgs and RHNs can not distinguish between the Majorana and pseudo-Dirac neutrinos, we use the same limits for both of cases. Similarly the EWPD can measure the deviation from the SM couplings so we can use the same bounds in both cases.

\section{Conclusions}

In this paper we have studied both type I and inverse Seesaw models where SM singlet RHNs are involved. The RHNs involved in type I Seesaw mechanism are Majorana type whereas those present in the inverse Seesaw model are pseudo-Dirac in nature. We have studied the production mechanisms of the RHNs from various initial states at the $13 \mathrm{TeV}$ LHC and future $100 \mathrm{TeV}$ pp collider considering the square of the mixing angle as $10^{-4}$ when the RHNs are produced in association with charged lepton and neutral lepton along with the jets. It has been shown due to the prompt decay of Higgs boson $\left(m_{N}<m_{H}\right)$ into the RHNs and SM light neutrino that the cross section dominates over all the remaining production modes. Such heavy neutrinos can dominantly decay into the $\ell W$ mode at the colliders. Depending on the nature of the model we pick up two distinguishing signals from type I Seesaw and inverse Seesaw in the form of same-sign dilepton plus di-jet and trilepton plus MET, respectively.

Using the recent searches by the CMS and ATLAS for type I Seesaw with same-sign di-muon and di-jet final state we put a prospective upper bound on $\left|V_{\mu N}\right|^{2}$ for the $13 \mathrm{TeV}$ LHC and future $100 \mathrm{TeV}$ pp collider at $3000 \mathrm{fb}^{-1}$ luminosity. Applying the cuts used in the anomalous multi-lepton search done by the LHC, we can put upper bounds on the square of the mixing angle between the SM light and of the degenerate RHNs. We consider the SF and FD cases where the limits in FD case are twice stronger than the SF case.

We have noticed that our currently given projected limits on the square of the mixing angles are better than those obtained from different experiments and even at the current stage of the LHC. We expect improvements in the status at the HL-LHC and future $100 \mathrm{TeV}$ pp collider when multilepton final states will be studied from the decays of the RHNs which will lead us to a more optimistic conclusion.

\section{Conflicts of Interest}

The author declares that there are no conflicts of interest regarding the publication of this paper.

\section{Acknowledgments}

The author would like to thank E. J. Chun, P. S. Bhupal Dev, Y. Gao, T. Kamon, P. Konar, R. N. Mohapatra, N. Okada, A. Thalapillil, and U. K. Yang for useful discussions at different times. The author would like to thank A. G. Hessler, A. Ibarra, E. Molinaro, and S. Vogl for useful information and discussions regarding [76].

\section{References}

[1] K. Abe et al., "Indication of Electron Neutrino Appearance from an Accelerator-Produced Off-Axis Muon Neutrino Beam," Physical Review Letters, vol. 107, 2011.

[2] P. Adamson, "Publisher's Note: Limits on Active to Sterile Neutrino Oscillations from Disappearance Searches in the MINOS, Daya Bay, and Bugey-3 Experiments," Physical Review Letters, vol. 107, no. 20, 2011.

[3] Abe Y. and et al, "Indication of Reactor $\bar{\nu}$ Disappearance in the Double Chooz Experiment," Physical Review Letters, vol. 108, Article ID 131801, 2012.

[4] F. P. An et al., "Observation of Electron-Antineutrino Disappearance at Daya Bay," Physical Review Letters, vol. 108, Article ID 171803, 2012.

[5] B. L. ROBERTS, "Observation of Reactor Electron Antineutrinos Disappearance in the RENO Experiment," Physical Review Letters, vol. 108, Article ID 191802, 2012.

[6] C. Patrignani et al., "[Particle Data Group], Review of Particle Physics," Chinese Physics C, vol. 10, Article ID 100001, 2016.

[7] S. Weinberg, "Baryon- and Lepton-nonconserving processes," Physical Review Letters, vol. 43, p. 1566, 1979.

[8] P. Minkowski, " $\mu \rightarrow \mathrm{e} \gamma$ at a rate of one out of $10^{9}$ muon decays?" Physics Letters B, vol. 67, pp. 421-428, 1977.

[9] T. Yanagida, "Horizontal Symmetry and Masses of Neutrinos," Progress of Theoretical and Experimental Physics, vol. 64, no. 3, pp. 1103-1105, 1980.

[10] J. Schechter and J. W. F. Valle, "Neutrino masses in $\mathrm{SU}(2) \otimes \mathrm{U}(1)$ theories," Physical Review D: Particles, Fields, Gravitation and Cosmology, vol. 22, p. 2227, 1980. 
[11] T. Yanagida, In Proceedings of the Work- Shop on the Unified Theory and the Baryon Number in the Universe, O. Sawada and A. Sugamoto, Eds., KEK, Tsukuba, Japan, 1979.

[12] M. Gell-Mann, P. Ramond, and R. Slansky, Supergravity, P. van Nieuwenhuizen et al., Ed., Amsterdam, Netherlands, 1979.

[13] S. L. Glashow, "The future of elementary particle physics," in Proceedings of the 1979 Cargese Summer Institute on Quarks and Leptons, M. Levy, Ed., p. 687, New York, 1980.

[14] R. N. Mohapatra and G. Senjanovic, "Neutrino mass and spontaneous parity nonconservation," Physical Review Letters, vol. 44, p. 912, 1980.

[15] J. A. Casas and A. Ibarra, "Oscillating neutrinos and $\mu \rightarrow e, \gamma$," Nuclear Physics B, vol. 618, no. 1-2, pp. 171-204, 2001.

[16] A. Das and N. Okada, "Bounds on heavy Majorana neutrinos in type-I seesaw and implications for collider searches," Physics Letters B, vol. 774, pp. 32-40, 2017.

[17] J. Adam et al., "New Limit on the Lepton-Flavor-Violating Decay $\mu^{+} \rightarrow \mathrm{e}^{+} \gamma$," Physical Review Letters, vol. 107, 2011.

[18] B. Aubert, Y. Karyotakis, and J. P. Lees, "Searches for Lepton flavor violation in the decays $\tau^{ \pm} \rightarrow e^{ \pm} \gamma$ and $\tau^{ \pm} \rightarrow \mu^{ \pm} \gamma$," Physical Review Letters, vol. 104, no. 2, Article ID 021802, 7 pages, 2010.

[19] B. O’Leary et al., "SuperB Progress Reports - Physics," https:// arxiv.org/abs/1008.1541.

[20] J. d. Blas, M. Bosman, A. Juste, M. Martínez, I. Riu, and V. Sorin, "Electroweak limits on physics beyond the Standard Mode," EPJ Web of Conferences, vol. 60, p. 19008, 2013.

[21] O. Adriani, L3 Collaboration et al., "Search for isosinglet neutral heavy leptons in $Z^{0}$ decays," Physics Letters B, vol. 295, pp. 371382, 1992.

[22] M. Acciarri et al., "Search for heavy isosinglet neutrinos in $\mathrm{e}^{+} \mathrm{e}^{-}$ annihilation at $130<\mathrm{s}<189 \mathrm{GeV}$," Physics Letters B, vol. 461, no. 4, pp. 397-404, 1999.

[23] P. Achard et al., "Search for heavy isosinglet neutrino in $\mathrm{e}^{+} \mathrm{e}^{-}$ annihilation at LEP," Physics Letters B, vol. 517, no. 1-2, pp. 6774, 2001.

[24] P. Abreu et al., "Search for neutral heavy leptons produced in $\mathrm{Z}$ decays," Zeitschrift für Physik, vol. 75, p. 57, 1997.

[25] A. Blondel, E. Graverini, N. Serra, and M. Shaposhnikov, "Search for Heavy Right Handed Neutrinos at the FCC-ee," Nuclear and Particle Physics Proceedings, vol. 273-275, pp. 18831890, 2016.

[26] F. del Aguila, J. de Blas, and M. Pérez-Victoria, "Effects of new leptons in electroweak precision data," Physical Review D: Particles, Fields, Gravitation and Cosmology, vol. 78, no. 1, 2008.

[27] E. Akhmedov, A. Kartavtsev, M. Lindner, L. Michaels, and J. Smirnov, "Improving electro-weak fits with $\mathrm{TeV}$-scale sterile neutrinos," Journal of High Energy Physics, vol. 2013, no. 5, article 081, 2013.

[28] S. Antush, C. Biggio, E. Fernandez-Martinez, M. Belen Gavela, and J. Lopez-Pavon, "Unitarity of the leptonic mixing matrix," Journal of High Energy Physics, vol. 2006, no. 10, p. 84, 2006.

[29] A. Abada, C. Biggio, F. Bonnet, M. B. Gavela, and T. Hambye, "Low energy effects of neutrino masses," Journal of High Energy Physics, vol. 2007, no. 12, article 061, 2007.

[30] A. Ibarra, E. Molinaro, and S. T. Petcov, "TeV scale see-saw mechanisms of neutrino mass generation, the Majorana nature of the heavy singlet neutrinos and $(\beta \beta) 0 \nu$-decay," Journal of High Energy Physics, vol. 2010, no. 9, article no. 108, 2010.

[31] A. Ibarra, E. Molinaro, and S. T. Petcov, "Low energy signatures of the TeV scale seesaw mechanism," Physical Review D:
Particles, Fields, Gravitation and Cosmology, vol. 84, Article ID 013005, 2011.

[32] D. N. Dinh, A. Ibarra, E. Molinaro, and S. T. Petcov, "Erratum: the $\mu$-e conversion in nuclei, $\mu \rightarrow \mathrm{e} \gamma, \mu \rightarrow 3$ e decays and $\mathrm{TeV}$ scale see-saw scenarios of neutrino mass generation," Journal of High Energy Physics, vol. 2013, no. 9, 2013.

[33] J. Penedo, S. Petcov, and T. T. Yanagida, "Low-scale seesaw and the CP violation in neutrino oscillations," Nuclear Physics B, 2018.

[34] R. W. Rasmussen and W. Winter, "Perspectives for tests of neutrino mass generation at the $\mathrm{GeV}$ scale: Experimental reach versus theoretical predictions," Physical Review D: Particles, Fields, Gravitation and Cosmology, vol. 94, no. 7, 2016.

[35] P. S. B. Dev, R. Franceschini, and R. N. Mohapatra, "Bounds on TeV seesaw models from LHC Higgs data," Physical Review D: Particles, Fields, Gravitation and Cosmology, vol. 86, no. 9, Article ID 093010, 2012.

[36] G. Aad et al., "Search for heavy Majorana neutrinos with the ATLAS detector in pp collisions at $\sqrt{s}=8 \mathrm{TeV}$," Journal of High Energy Physics, vol. 162, 2015.

[37] V. Khachatryan, " Erratum: Search for single production of scalar leptoquarks in proton-proton collisions at ", Physical Review D: Particles, Fields, Gravitation and Cosmology, vol. 95, no. 3, 2017.

[38] J. Kersten and A. Y. Smirnov, "Right-handed neutrinos at LHC and the mechanism of neutrino mass generation," Physical Review D: Particles, Fields, Gravitation and Cosmology, vol. 76, Article ID 073005, 2007.

[39] Z. Xing, "Naturalness and Testability of TeV Seesaw Mechanisms," Progress of Theoretical and Experimental Physics Supplement, vol. 180, pp. 112-127, 2009.

[40] X. He, S. Oh, J. Tandean, and C. Wen, "Large mixing of light and heavy neutrinos in seesaw models and the LHC," Physical Review D: Particles, Fields, Gravitation and Cosmology, vol. 80, no. 7, 2009.

[41] F. F. Deppisch and A. Pilaftsis, "Lepton Flavour Violation and theta(13) in Minimal Resonant Leptogenesis," Physical Review $D$, vol. 83, Article ID 076007, 2011.

[42] C.-H. Lee, P. S. B. Dev, and R. N. Mohapatra, "Natural TeV-scale left-right seesaw mechanism for neutrinos and experimental tests," Physical Review D: Particles, Fields, Gravitation and Cosmology, vol. 88, no. 9, Article ID 093010, 2013.

[43] R. N. Mohapatra, "Mechanism for understanding small neutrino mass in superstring theories," Physical Review Letters, vol. 56 , no. 6, pp. 561-563, 1986.

[44] R. N. Mohapatra and J. W. F. Valle, "Neutrino mass and baryonnumber nonconservation in superstring models," Physical Review D: Particles, Fields, Gravitation and Cosmology, vol. 34, no. 5, pp. 1642-1645, 1986.

[45] A. Das, P. B. Dev, and C. Kim, "Constraining sterile neutrinos from precision Higgs data," Physical Review D: Particles, Fields, Gravitation and Cosmology, vol. 95, no. 11, 2017.

[46] A. Das, Y. Gao, and T. Kamon, Heavy Neutrino Search via the Higgs boson at the LHC, arXiv, 1704.00881, arXiv:1704.00881.

[47] A. Das and N. Okada, "Inverse seesaw neutrino signatures at the LHC and ILC," Physical Review D: Particles, Fields, Gravitation and Cosmology, vol. 88, no. 11, Article ID 113001, 2013.

[48] A. Das, P. Bhupal Dev, and N. Okada, "Direct bounds on electroweak scale pseudo-Dirac neutrinos from $\sqrt{s}=8 \mathrm{TeV}$ LHC data," Physics Letters B, vol. 735, pp. 364-370, 2014. 
[49] A. Das and N. Okada, "Improved bounds on the heavy neutrino productions at the LHC," Physical Review D: Particles, Fields, Gravitation and Cosmology, vol. 93, no. 3, 2016.

[50] A. Das, N. Nagata, and N. Okada, "Testing the $2-\mathrm{TeV}$ resonance with trileptons," Journal of High Energy Physics, vol. 2016, no. 3, 2016.

[51] A. Das, P. Konar, and S. Majhi, "Production of heavy neutrino in next-to-leading order QCD at the LHC and beyond," Journal of High Energy Physics, vol. 2016, no. 6, 2016.

[52] A. Das, "Pair production of heavy neutrinos in next-to-leading order QCD at the hadron colliders in the inverse Seesaw framework," https://arxiv.org/abs/1701.04946.

[53] N. Arkani-Hamed, T. Han, M. Mangano, and L.-T. Wang, "Physics opportunities of a $100 \mathrm{TeV}$ proton-proton collider," Physics Reports, vol. 652, pp. 1-49, 2016.

[54] J. Alwall, M. Herquet, F. Maltoni, O. Mattelaer, and T. Stelzer, "MadGraph 5: going beyond," Journal of High Energy Physics, vol. 128, 2011.

[55] T. Sjostrand, S. Mrenna, and P. Skands, "PYTHIA 6.4 physics and manual," Journal of High Energy Physics, vol. 5, article 026, 2006.

[56] J. de Favereau, C. Delaere, P. Demin et al., "DELPHES 3: a modular framework for fast simulation of a generic collider experiment," Journal of High Energy Physics, vol. 2014, article 57, 2014.

[57] S. Catani, F. Krauss, B. R. Webber, and R. Kuhn, "QCD Matrix Elements + Parton Showers," Journal of High Energy Physics, vol. 2001, no. 11, pp. 063-063, 2001.

[58] J. Alwal, "MadGraph Wiki," Matching of Jets between MadFevent and Pythia, p. 10, 2011, https://cp3.irmp.ucl.ac.be/projects/madgraph/wiki/Matching.

[59] J. Alwall and S. D. Visscher, "Introduction to jet-parton matching in MG/ME," MadGraph Wiki, 2011, https://cp3.irmp .ucl.ac.be/projects/madgraph/wiki/IntroMatching.

[60] J. Alwall, "IPMU," in Proceedings of the Focus week, Tokyo, Japan, http://www.ipmu.jp/sites/default/files/webfm/pdfs/FocusWeek_10.

[61] J. Alwall, Parton Showers and MLM matching with MadGraph and Pythia, NTU-MadGraph School, 2012, https://cp3.irmp .ucl.ac.be/projects/madgraph/attachment//NTU-MLM-lectures.pdf.

[62] J. Alwall, "HELAS/MadGraph Workshop," KEK, pp. 1827, 2006, http://phya.snu.ac.kr/ particle/wp-content/uploads/ 2007/03/lectureb-1.pdf.

[63] J. Alwall, "HELAS/MadGraph Workshop," KEK, 2006, http:// phya.snu.ac.kr/ particle/wp-content/uploads/2007/03/lectureb-2.pdf.

[64] J. Alwall, "HELAS/MadGraph Workshop," KEK, pp. 1827, 2006, http://phya.snu.ac.kr/ particle/wp-content/uploads/ 2007/03/lectureb-3.pdf.

[65] M. L. Mangano, F. Piccinini, A. D. Polosa, M. Moretti, and R. Pittau, http://mlm.web.cern.ch/mlm/talks/kek-alpgen.pdf, http://mlm.home.cern.ch/mlm/alpgen.

[66] M. L. Mangano, F. Piccinini, A. D. Polosa, M. Moretti, and R. Pittau, "ALPGEN, a generator for hard multiparton processes in hadronic collisions," Journal of High Energy Physics, vol. 2003, no. 07, pp. 001-001, 2003.

[67] M. L. Mangano, M. Moretti, F. Piccinini, and M. Treccani, "Matching matrix elements and shower evolution for top-pair production in hadronic collisions," Journal of High Energy Physics, vol. 2007, no. 1, article no. 013, 2007.
[68] B. Cooper, J. Katzy, M. L. Mangano, A. Messina, L. Mijović, and P. Skands, "Importance of a consistent choice of $\alpha \mathrm{S}$ in the matching of AlpGen and Pythia," The European Physical Journal C, vol. 72, no. 7, 2012.

[69] J. Alwall, S. Höche, F. Krauss et al., "Comparative study of various algorithms for the merging of parton showers and matrix elements in hadronic collisions," The European Physical Journal C, vol. 53, no. 3, pp. 473-500, 2008.

[70] J. Alwall, S. d. Visscher, and F. Maltoni, "QCD radiation in the production of heavy colored particles at the LHC," Journal of High Energy Physics, vol. 2009, no. 02, pp. 017-017, 2009.

[71] http://home.thep.lu.se/ torbjorn/pythia81html/JetMatching .html.

[72] http://www.tifr.res.in/ indiacms/indiacms-meetings/march2009/Matching\%20Parton\%20Showers\%20and\%20Matrix\%20 elements.pdf.

[73] S. Hoeche, F. Krauss, N. Lavesson et al., Matching parton showers and matrix elements, Particle Physics - Phenomenology, CERN Document Server, 2005.

[74] S. Chatrchyan et al., "Search for anomalous production of events with three or more leptons in pp collisions at $\sqrt{ } \mathrm{s}=8 \mathrm{TeV}$," Physical Review D, vol. 90, 2014.

[75] The CMS collaboration, S. Chatrchyan et al., "Search for anomalous production of multilepton events in pp collisions at $\sqrt{s}=7 \mathrm{TeV}$,' Journal of High Energy Physics, 2012.

[76] A. G. Hessler, A. Ibarra, E. Molinaro, and S. Vogl, "Impact of the Higgs boson on the production of exotic particles at the LHC," Physical Review D: Particles, Fields, Gravitation and Cosmology, vol. 91, no. 11, Article ID 115004, 2015.

[77] R. Ruiz, M. Spannowsky, and P. Waite, "Heavy neutrinos from gluon fusion," Physical Review D: Particles, Fields, Gravitation and Cosmology, vol. 96, no. 5, 2017.

[78] E. J. Williams, "Nature of the high energy particles of penetrating radiation and status of ionization and radiation formulae," Physical Review A: Atomic, Molecular and Optical Physics, vol. 45, p. 729, 1934.

[79] F. C. von Weizsacker, "Radiation emitted in collisions of very fast electrons," Zeitschrift für Physik, vol. 88, p. 612, 1934.

[80] V. M. Budnev, I. F. Ginzburg, G. V. Meledin, and V. G. Serbo, "The two-photon particle production mechanism. Physical problems. Applications. Equivalent photon approximation," Physics Reports, vol. 15, no. 4, pp. 181-282, 1975.

[81] P. S. B. Dev, A. Pilaftsis, and U.-K. Yang, "New production mechanism for heavy neutrinos at the LHC," Physical Review Letters, vol. 112, Article ID 081801, 2014.

[82] D. Alva, T. Han, and R. Ruiz, "Heavy Majorana neutrinos from W $\gamma$ fusion at hadron colliders," Journal of High Energy Physics, vol. 2015, no. 2, 2015.

[83] F. F. Deppisch, P. S. B. Dev, and A. Pilaftsis, "Neutrinos and collider physics," New Journal of Physics, vol. 17, no. 7, Article ID 075019, 2015.

[84] M. Drewes, "The phenomenology of right handed neutrinos," International Journal of Modern Physics E, vol. 22, no. 8, Article ID 1330019, 2013.

[85] S. Dube, D. Gadkari, and A. M. Thalapillil, "Lepton jets and low-mass sterile neutrinos at hadron colliders," Physical Review D: Particles, Fields, Gravitation and Cosmology, vol. 96, no. 5, Article ID 055031, 2017.

[86] A. Das, P. B. Dev, and R. N. Mohapatra, "Same sign versus opposite sign dileptons as a probe of low scale seesaw mechanisms," Physical Review D: Particles, Fields, Gravitation and Cosmology, vol. 97, no. 1, 2018. 
[87] A. Das, P. Konar, and A. Thalapillil, "Jet substructure shedding light on heavy Majorana neutrinos at the LHC," Journal of High Energy Physics, vol. 2018, no. 2, 2018.

[88] A. Bhardwaj, A. Das, P. Konar, and A. Thalapillil, "Challenging Sterile Neutrino Searches at the LHC Complemented by Jet Substructure Techniques".

[89] A. Das, N. Okada, and D. Raut, "Enhanced pair production of heavy Majorana neutrinos at LHC," https://arxiv.org/abs/ 1710.03377 .

[90] A. Das, N. Okada, and D. Raut, "Heavy Majorana neutrino pair productions at the LHC in minimal U(1) extended Standard Model," https://arxiv.org/abs/1711.09896.

[91] M. Mitra, R. Ruiz, D. J. Scott, and M. Spannowsky, "Neutrino jets from high-mass WR gauge bosons in TeV-scale leftright symmetric models," Physical Review D: Particles, Fields, Gravitation and Cosmology, vol. 94, no. 9, Article ID 095016, 2016.

[92] D. Das, K. Ghosh, M. Mitra, and S. Mondal, "Probing sterile neutrinos in the framework of inverse seesaw mechanism through leptoquark productions," Physical Review D: Particles, Fields, Gravitation and Cosmology, vol. 97, no. 1, 2018.

[93] M. Drewes, B. Garbrecht, D. Gueter, and J. Klarić, “Testing the low scale seesaw and leptogenesis," Journal of High Energy Physics, vol. 2017, no. 8, 2017.

[94] Y. L. Tang, "Probing the Light Sterile Neutrino Through the Heavy Charged Higgs Decay on the LHC," https://arxiv.org/ abs/1712.10108.

[95] S. Chatrchyan et al., "Search for the standard model Higgs boson decaying to $\mathrm{W}+\mathrm{W}$ - in the fully leptonic final state in $\mathrm{pp}$ collisions at $\sqrt{s}=8 \mathrm{TeV}$," Physics Letters B, vol. 710, p. 91, 2012, arXiv:1202.1489.

[96] CMS Collaboration, "Search for the standard model Higgs boson decaying to a $\mathrm{W}$ pair in the fully leptonic final state in pp collisions at $\sqrt{s}=8 \mathrm{TeV}$," CMS-PAS-HIG.

[97] G. Aad et al., "Search for the Standard Model Higgs boson in the $H \rightarrow W W^{(*)} \rightarrow \ell v \ell v>$ decay mode with $4.7 \mathrm{fb}$ of ATLAS data at $\sqrt{s}=7$ TeV," Physics Letters B, vol. 716, pp. 62-81, 2012.

[98] S. Chatrchyan et al., "Search for the standard model Higgs boson in the $H \rightarrow Z Z \rightarrow 2 \ell 2 \nu$ channel inin pp collisions at $\sqrt{s}=7$ TeV," Journal of High Energy Physics, vol. 1203, 2012.

[99] J. Meyer et al., "Search for a standard sodel Higgs boson in the $H \rightarrow Z Z \rightarrow \ell^{+} \ell^{-} \bar{v}$ decay channel using $4.7 \mathrm{fb}-1$ of $\sqrt{s}=7 \mathrm{TeV}$ data with the ATLAS detector," Physics Letters B, vol. 29, 2012.

[100] J. Pumplin, D. R. Stump, J. Huston et al., "New Generation of Parton Distributions with Uncertainties from Global QCD Analysis," Journal of High Energy Physics, vol. 020, p. 7, 2002.

[101] V. Khachatryan et al., "Search for heavy Majorana neutrinos in $\mu \pm \mu \pm+$ jets events in proton-proton collisions at $\sqrt{s}=8 \mathrm{TeV}$," in Physics Letters B, vol. 748, pp. 144-166. 

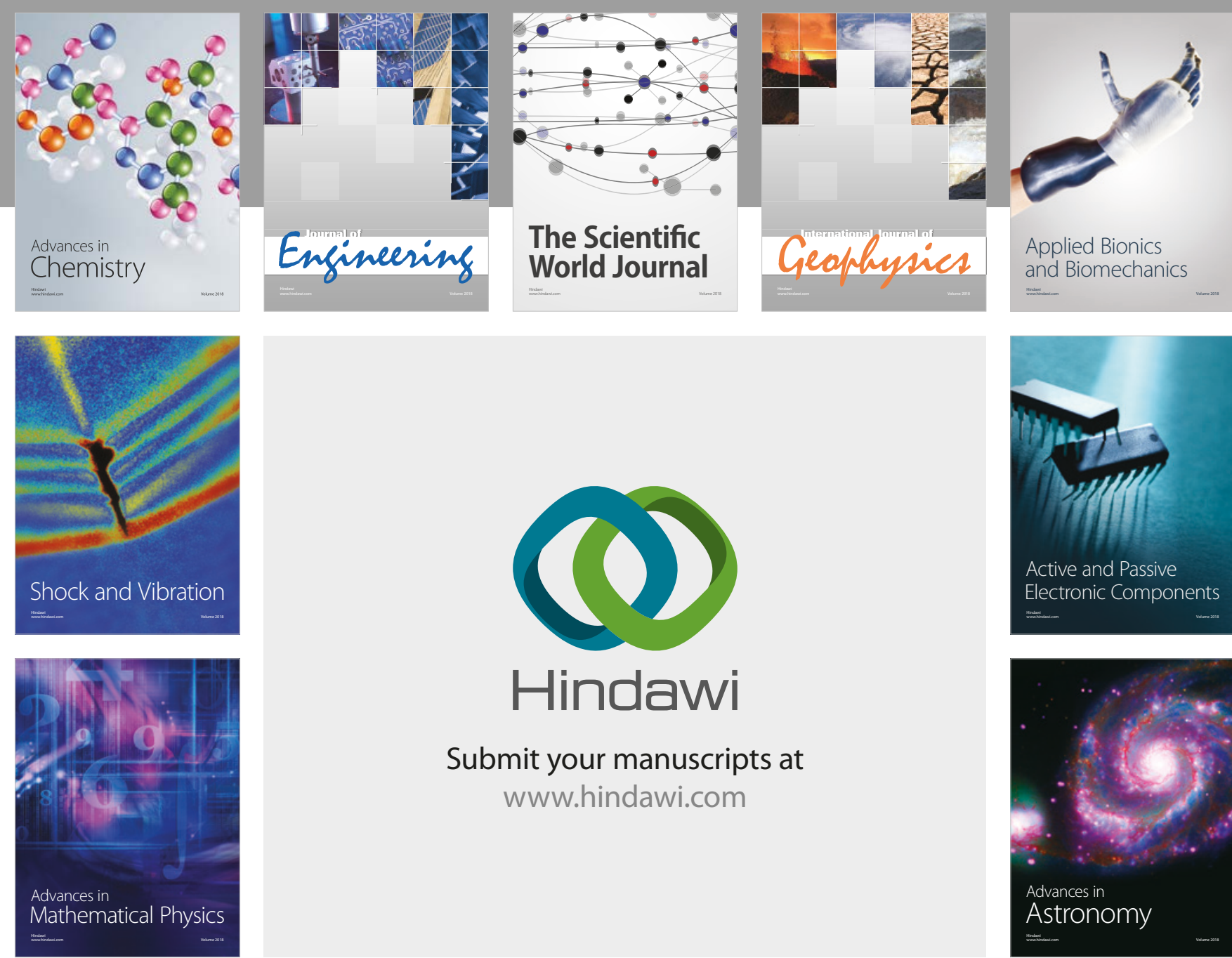

Submit your manuscripts at

www.hindawi.com

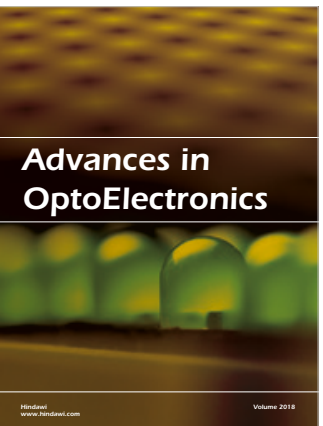

\section{Rotcting Machinery}
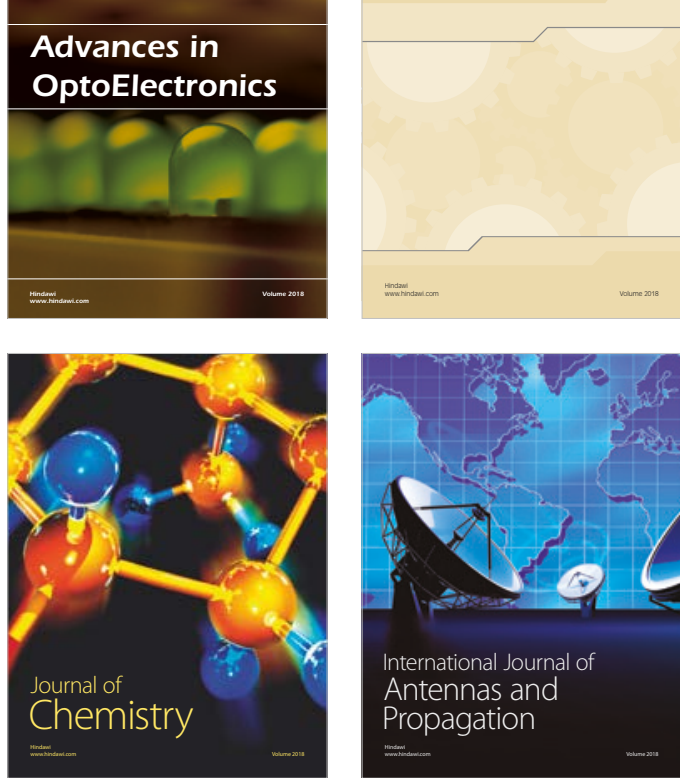

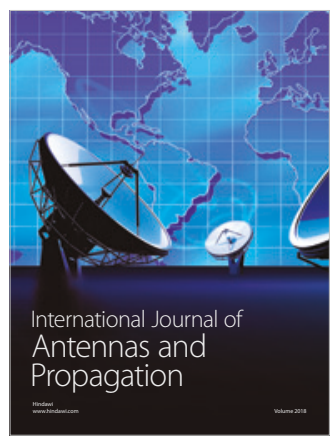

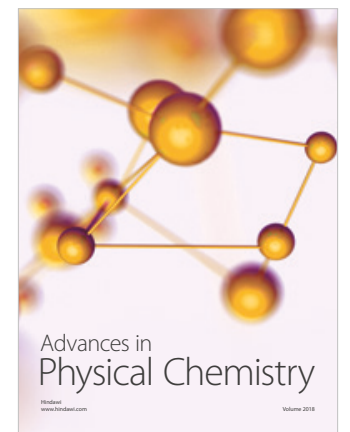

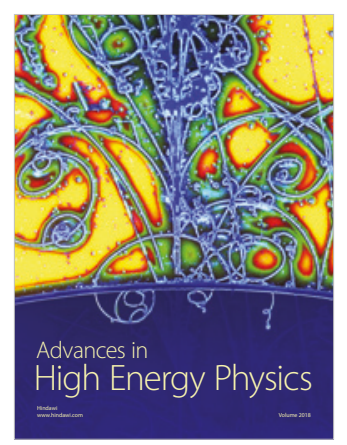

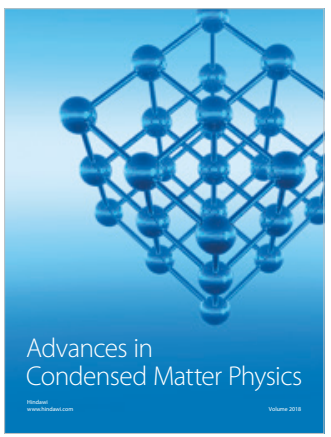

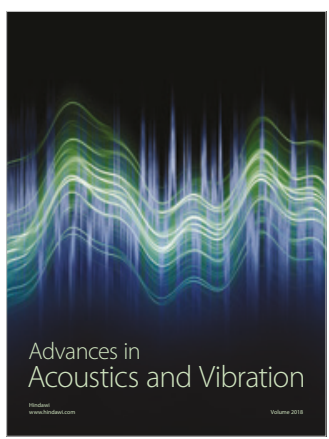

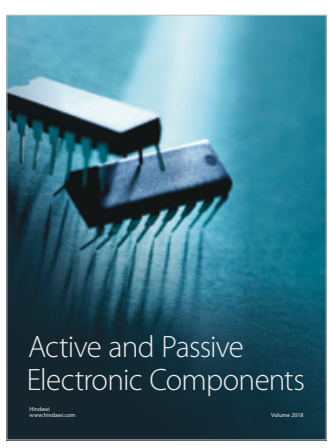
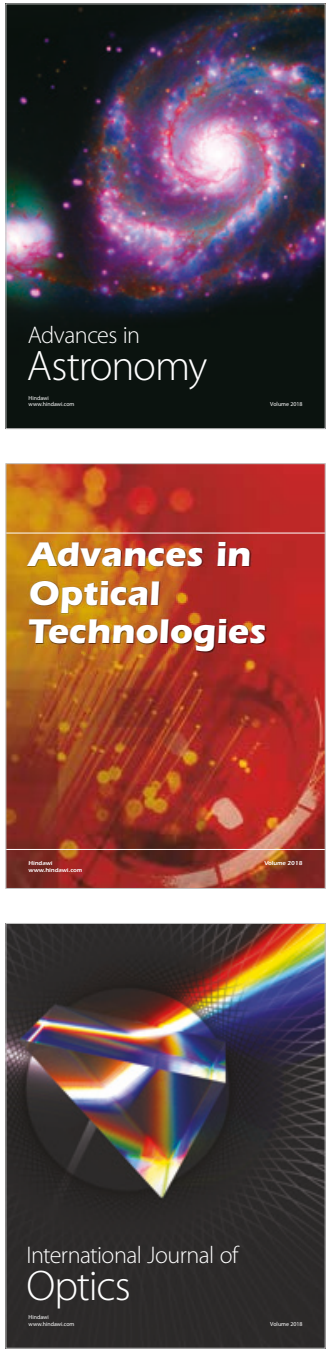